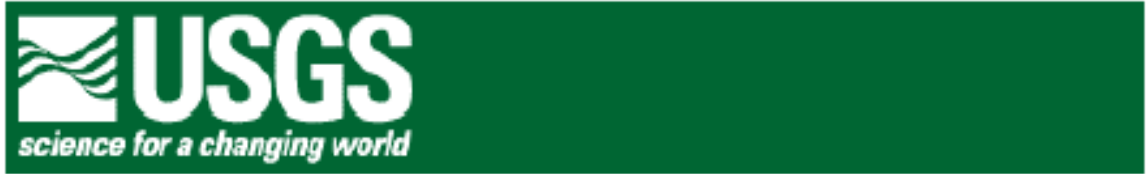

\title{
ELECTRICAL PROPERTY MEASUREMENTS OF MINE WASTE FROM THE SUNDAY \#2 AND VENIR MINES, LEADVILLE, COLORADO
}

by Robert J. Horton

Denver, Colorado

\section{Open-File Report 03-149} 2003

This report is preliminary and has not been reviewed for conformity with U.S. Geological

Survey editorial standards and stratigraphic nomenclature. Any use of trade, product, or firm names is for descriptive purposes only and does not imply endorsement by the U.S. Government.

\section{U.S. DEPARTMENT OF THE INTERIOR U.S. GEOLOGICAL SURVEY}




\section{Introduction}

This report describes the results of electrical property measurements made on samples of mine waste collected from the Sunday \#2 and Venir mine sites located in the Leadville Mining District. These laboratory measurements were made as part of the USGS Mineral Resource Program, Mine Waste Characterization project, which consists of geological, geochemical, and geophysical studies of abandoned mine lands in Colorado and New Mexico. These laboratory measurements were made to look for correlations between the geochemical characteristics and electrical properties of mine waste. Knowledge of the waste's electrical properties is also useful in the design and interpretation of field-scale electrical and electromagnetic geophysical surveys.

The Leadville Mining District is on the western slope of the Mosquito Range in the Central Colorado Rocky Mountains. Mining and milling activities have produced sulfide-rich waste that now covers approximately $30 \mathrm{~km}^{2}$ of the district. The Venir and Sunday \#2 mines are located on the west flank of Ball Mountain, east of Leadville, Colorado (Figure 1). The Venir mine dump is located at $\mathrm{N} 39^{\circ} 14^{\prime} 18^{\prime \prime}, \mathrm{W} 106^{\circ} 14^{\prime} 1^{\prime \prime}$ at an elevation of about $11695 \mathrm{ft}$, whereas the Sunday \#2 dump is located at N39 ${ }^{\circ} 14^{\prime} 07^{\prime \prime}, \mathrm{W} 106^{\circ} 13^{\prime} 40^{\prime \prime}$ at an elevation of about $11940 \mathrm{ft}$.

For this laboratory study, the electrical properties were measured using the complex resistivity (CR) method, also known as spectral induced polarization. The laboratory instrumentation measures the sample's resistivity and phase as a function of frequency. Resistivity and phase are complex quantities, having both real and imaginary components, hence the method's name. CR measurements are made by transmitting electric current through the sample and measuring a received voltage. The current is transmitted as a sine wave and measurements are made over a wide band of discrete frequencies. CR data are plotted as resistivity and phase spectra, which show the frequency dependency of the measured electrical properties.

\section{Resistivity}

Resistivity is a measure of a material's opposition to the flow of electric current. Resistivity is the inverse of electric conductivity, the ability to transport electric charge. Resistivity units are ohm meters (ohm-m). The resistivity of the mine waste is dependent on several factors including the amount of water present, porosity, the amount of total dissolved solids (TDS) in pore water, and the mineral composition of the waste. The amount and TDS of pore water are typically the dominant factors that determine a samples resistivity. All other factors being equal, dry waste is more resistive than saturated waste and waste saturated with low TDS water is more resistive than waste saturated with high TDS water. 


\section{Phase}

Phase is the angle of lag, or lead, of a sine wave with respect to a reference. Phase units are in milliradians ( $\mathrm{mRad}$ ). Phase is a measure of charge storage or polarization. For these measurements the phase of the received voltage is measured using the current wave form as the reference. When electric current is transmitted through a material, a temporary induced polarization effect is generated. Induced polarization is a redistribution of charged particles, such as ions and electrons, from their equilibrium position due to the applied current. This redistribution or storage of charge is not an instantaneous process, but requires a finite period to develop and to dissipate as the current varies. The time required to develop and dissipate the stored charge produces the phase lag observed between the received voltage and the transmitted current.

\section{Frequency Dependency}

Frequency dependency results from different mechanisms of charge transport and charge storage. Some of these mechanisms operate rapidly and are observed at relatively high frequencies, whereas slow mechanisms are observed at lower frequencies. At low frequencies, the electrical properties of rocks and minerals are dominated by charge transport or conduction mechanisms, whereas at high frequencies charge storage or polarization mechanisms dominate.

A quantitative measure of frequency dependent resistivity, historically used to interpret induced polarization measurements, is the percent frequency effect (PFE) defined as:

$$
\text { PFE }=100\left(\rho_{\text {low }}-\rho_{\text {high }}\right) / \rho_{\text {high }}
$$

Where:

$$
\begin{aligned}
& \rho_{\text {low }}=\text { low frequency resistivity } \\
& \rho_{\text {high }}=\text { high frequency resistivity }
\end{aligned}
$$

For this report, PFE values were calculated using data from $0.1 \mathrm{~Hz}$ and $1.0 \mathrm{~Hz}$.

\section{Linearity}

The electrical properties of most minerals are linear. A sample exhibits linear behavior if its electrical properties are independent of the amplitude of the applied electric current. For example, if a sample's resistivity or phase remains constant as the input current is varied, it has linear properties because the received voltage varies proportionally with the current. However, some minerals do exhibit non-linear behavior. Non-linear behavior is observed in minerals actively involved in oxidation-reduction reactions and ion exchange processes (Olhoeft, 1979).

The electrical data in this report has been analyzed using two measures of linearity including Hilbert distortion and total harmonic distortion. These 
measures of linearity were calculated using the method given by Olhoeft (1985) applied in a program written by Jones (1997). The real and imaginary parts of the complex resistivity expression are related through the Hilbert transform. By applying the Hilbert transform to the measured resistivity and phase, a "Hilbert resistivity" and "Hilbert phase" can be calculated. Hilbert distortion (HD) is the difference between the measured resistivity and phase and the calculated resistivity and phase derived from the Hilbert transform. In a linear system the received signal has the same harmonic content as the transmitted signal. The total harmonic distortion (THD) value is the root-mean-square difference between the harmonic content of the transmitted and received wave forms.

The type of non-linearity exhibited by a sample provides information about electrochemical processes within the sample. In general, the non-linearity exhibited by the oxidation-reduction of sulfide minerals is expressed as total harmonic distortion, whereas cation-exchange reactions involving clay minerals produce Hilbert distortion. However, both oxidation-reduction and cation exchange reactions can behave in a linear fashion depending on the current density applied to the system. See Olhoeft (1979 and 1985) for additional information on non-linear electrical properties.

\section{Instrumentation}

The electrical properties presented here were measured using the nonlinear complex resistivity (NLCR) impedance spectroscopy instrumentation in the Geophysics Department, Petrophysics Laboratory at the Colorado School of Mines. This instrumentation was assembled by Jones (1997), and is a modification of the instrumentation described by Olhoeft (1985). The NLCR instrumentation measures electrical properties over a wide band of frequencies ranging from $2 \times 10^{-3}$ to $10^{6} \mathrm{~Hz}$ (two millihertz to 1 megahertz) with three discrete frequencies being measured per decade.

Samples were measured in a cylindrical sample holder made of white virgin Teflon. The sample occupies a space 1 inch in diameter and three inches long, having a volume of 2.356 cubic inches $\left(38.62 \mathrm{~cm}^{3}\right)$. Electrical signals were transmitted to, and received from, the sample through four equally spaced electrodes made from bright platinum mesh. Two electrodes at the ends of the sample holder were used to transmit current through the sample. The voltage response of the sample was measured across two electrodes spaced 1 inch apart in the center of the sample holder. An in depth description of the laboratory procedures and apparatus can be found in Campbell and Horton (2000).

\section{Sample Preparation}

Six samples were measured for this report. Each sample was measured a number of times with different water weight percents. These samples were collected from distinct mineralogical zones observed at the sites for spectroscopic and mineral analysis (Swayze and others, 2000). The mineralogical analysis of the samples are given in Table 1. 
The measured samples are splits of larger samples collected from shallow surfacial material from less than $2 \mathrm{~cm}$ deep. The samples had been sieved to $<4.0 \mathrm{~mm}$ and were dry, no attempt was made to preserve their original moisture content. Typically, samples are first measured in their "as received" condition, however, as received the Leadville samples were too resistive to measure. Therefore, a small amount of laboratory grade, deionized water was added to each sample to lower its' resistivity.

The amount of water initially added to the sample was 3 to 4 percent by weight. The mixture was thoroughly stirred then loaded into the sample holder for measurement. For subsequent measurements, more water was added until saturated. Water weight percent was determined by weight and sample density was calculated using the sample holder volume. Because the sample, as received, may not have been completely dry, the calculated water weight percent given in table 2 is only approximate.

\section{Results}

The results of each electrical property measurement are presented on three panels that give different properties as a function of frequency (figure 2). The horizontal axis of each panel is logarithmic scaled frequency. The top panel gives the resistivity and phase spectra with resistivity plotted as triangular symbols (left legend, linear scale) and phase plotted as circle or diamond symbols (right legend, logarithmic scale). Phase values are normally negative and are plotted as a circle. However, if the phase is positive, unusual but possible, the sign of the value is changed and it is plotted as a diamond. The vertical whisker through each symbol indicates measurement error. The middle panel gives percent Hilbert distortion in the resistivity measurement (\%RHD, triangle symbols, left legend) and Hilbert distortion in the phase measurement (PHD/mR, circle symbols, right legend). The bottom panel give percent total harmonic distortion (\%THD).

Two basic spectral shapes were observed for most of the measurements For the purposes of the following discussion, these two shapes will be referred to as type A and B. Type A resistivity spectra show two breaks in slope, at low- and high-frequencies, with associated phase dispersions. Sample LV-1A2B has type A spectra (figures 11-12). The low-frequency break, typically between 0.01 and $1.0 \mathrm{~Hz}$, is associated with a broad, moderate-amplitude (>100 mRad) dispersion in the phase spectra. The high-frequency resistivity break, typically above $10,000 \mathrm{~Hz}\left(10^{4} \mathrm{~Hz}\right)$ is associated with a high-amplitude $(>1000 \mathrm{mRad})$ dispersion.

Type B resistivity spectra show three breaks in slope, at low-, middle- and high-frequencies, with associated dispersions in the phase spectra. Sample LV$1 \mathrm{G} 2 \mathrm{H}$ has type $\mathrm{B}$ spectra (figures 2-5). The low-frequency break, typically between 0.01 and $0.1 \mathrm{~Hz}$, is associated with a broad, low-amplitude (1-10 mRad) 
dispersion in the phase spectra. The middle resistivity break, typically between 100 and $1000 \mathrm{~Hz}$, is associated with a narrow, moderate-amplitude (>10 mRad) phase dispersion. The high-frequency resistivity break, typically above 100,000 $\mathrm{Hz}\left(10^{5} \mathrm{~Hz}\right)$ is associated with a high-amplitude (>1000 mRad) dispersion.

Following are the resistivity and phase spectra for each sample measurement and a description that includes the sample location and mineral analysis. For each measurement or "run", the low-frequency resistivity, frequency dependency, and linearity are described. The low-frequency resistivity value is an important measurement as it best approximates the resistivity of the material had it been measured in-situ using direct current (DC) resistivity techniques. For this report, the low-frequency measurement was usually made at $0.002 \mathrm{~Hz}$. Lowfrequency resistivity values are given in table 2 , and PFE values are given in table 3.

\section{$\underline{\mathrm{LV}-1 \mathrm{G} 2 \mathrm{H}}$}

This sample was collected from the jarosite+goethite zone at the Venir site. XRD analysis indicates the sample contains major quartz; minor jarosite, montmorillonite, orthoclase, muscovite, and pyrite; and trace copiapite and kaolinite. the sample was measured 4 different times. The first measurement (figure 2) had a water content of 17.3 weight percent, and a resistivity of $6.3 \mathrm{ohm}-$ $\mathrm{m}$. The second (figure 3 ) and third (figure 4) runs were repeat measurements made 30 and 60 minutes after the first measurement. Both the second and third runs had resistivities of about $6.1 \mathrm{ohm}-\mathrm{m}$. For the fourth run, the sample was allowed to sit overnight. The fourth run (figure 5 ) had a water content of $14.0 \%$, and a resistivity of $5.5 \mathrm{ohm}-\mathrm{m}$.

All the LV-IG2H runs have similar type B resistivity and phase spectra. All the spectra exhibit a high-frequency dielectric dispersion. The resistivity spectra also shows two regions of strong frequency dependency, one at frequencies less than $0.1 \mathrm{~Hz}$, and another centered at about $100 \mathrm{~Hz}$. The phase spectra show two dispersions, one broad low-frequency dispersion, and a narrower dispersion centered at about $100 \mathrm{~Hz}$. The PFE values range from 1.8 to $2.2 \%$, and decrease with each successive measurement.

\section{$\underline{L V-1 W X}$}

This sample was collected from the Venir site. XRD analysis indicates the sample contains major quartz; minor jarosite, muscovite, and pyrite; and trace orthoclase, copiapite and kaolinite. LV-1WX was measured 5 different times. The first run (figure 6 ) had a water content of 3.7 weight percent. The second run (figure 7) was a repeat measurement 30 minutes later. For the first and second runs, the low-frequency resistivity of the sample was 429 and 422 ohm-m respectively. Water was added for the third run (figure 8 ), increasing the water content to $15.6 \%$. For the third run, the resistivity decreased to $5.7 \mathrm{ohm}-\mathrm{m}$. The sample was allowed to sit overnight before the fourth and fifth runs. For the fourth run (figure 9 ) the water content was $9.7 \%$ and the resistivity decreased to 4.6 
ohm-m. The fifth run (figure 10) was a repeat measurement using a smaller volume sample holder, and gave a resistivity of $4.3 \mathrm{ohm}-\mathrm{m}$.

The first two measurements have similar type A spectra that show a strong frequency dependent resistivity at frequencies less than $0.5 \mathrm{~Hz}$. This frequency dependency is associated with a broad phase dispersion centered at about $0.1 \mathrm{~Hz}$. The last three measurements have similar type B spectra. The resistivity spectra shows two regions of strong frequency dependency, one at frequencies less than $0.1 \mathrm{~Hz}$, and another centered at about $20 \mathrm{~Hz}$. The phase spectra show two dispersions, including a broad low-frequency dispersion and a narrow dispersion centered at about $200 \mathrm{~Hz}$. The PFE values range from 1.9 to $21.1 \%$. The PFE values decrease as water was added with each successive measurement. The dry measurements have PFE values about an order of magnitude higher than the wet measurements.

\section{$\mathrm{LV}-1 \mathrm{~A} 2 \mathrm{~B}$}

This sample was collected from the Venir site. XRD analysis indicates the sample contains major quartz; minor jarosite, muscovite, and pyrite; and trace orthoclase, montmorillonite, copiapite and kaolinite. The sample was only measured twice. The first run (figure 11) had a water content of 3.4 weight percent. The sample was allowed to sit overnight before the second run. For the second run (figure 12) the water content was $5.4 \%$, and a resistivity of $15.3 \mathrm{ohm}-$ $\mathrm{m}$.

The LV-1A2B spectra are type A. The first run shows a high-frequency dielectric dispersion, and strong frequency dependency at frequencies less than $0.1 \mathrm{~Hz}$ that are associated with a broad phase dispersion. The center of the phase dispersion appears to be "off the chart" at a frequency below $0.001 \mathrm{~Hz}$. The second run shows a high-frequency dielectric dispersion, and strong frequency dependency at frequencies less than $0.1 \mathrm{~Hz}$. There is a broad phase dispersion that appears to be centered "off the chart" at a frequency below 0.001 $\mathrm{Hz}$. The PFE values, for the first and second measurements, are 12.6 and $7.1 \%$ respectively.

\section{$\underline{L V-2 W X}$}

This sample was collected from the goethite zone of the Sunday \#2 site. XRD analysis indicates the sample contains major quartz; minor muscovite; and trace orthoclase, jarosite and kaolinite. Sample LV-2WX as measured 4 different times. The first run (figure 13) had a water content of 3.8 weight percent, and a resistivity of $4895 \mathrm{ohm}-\mathrm{m}$. Water content was increased to $11.7 \%$ for the second run (figure 14), and the resistivity was $165 \mathrm{ohm}-\mathrm{m}$. The sample was allowed to sit overnight before the third and fourth runs. For the third run (figure 15) the water content was $9.8 \%$, the resistivity was $658 \mathrm{ohm}-\mathrm{m}$. For the fourth run (figure 16) the water content was increased to $19.2 \%$, resulting in a slurry-like consistency with a resistivity of $51.9 \mathrm{ohm}-\mathrm{m}$. 
The first measurement has a type A spectra showing a high-frequency dispersion due to dielectric effects, and a low-frequency dispersion centered at about $0.1 \mathrm{~Hz}$.. The second run shows a high-frequency dielectric dispersion and a low-amplitude dispersion centered at about $100 \mathrm{~Hz}$. The second run spectra is neither type A or B, as it does not have a low-frequency dispersion. The third measurement (figure 15) has a type A spectra with a high-frequency dielectric dispersion and a broad, low-frequency dispersion centered at $1.0 \mathrm{~Hz}$. The spectra shows the resistivity to be strongly frequency dependent below $1.0 \mathrm{~Hz}$. The fourth run (figure 16) shows a high-frequency dielectric dispersion and a minor dispersion centered at $100 \mathrm{~Hz}$. The resistivity data show almost no frequency dependency at frequencies below $1000 \mathrm{~Hz}$. The fourth run spectra is neither type A or B, as it does not have a low-frequency dispersion. PFE values range from 0.1 to $32.3 \%$.

\section{$\underline{L V-2 Q R}$}

This sample was collected from the jarosite zone of the Sunday \#2 site. XRD analysis indicates the sample contains major quartz; minor jarosite, muscovite, and illite; and trace pyrite and kaolinite. The sample was measured 3 different times. The first run (figure 17) had a water content of 3.7 weight percent, and a resistivity of $1142 \mathrm{ohm}-\mathrm{m}$. The water content was increased to $22.5 \%$ for the second run (figure 18), resulting in a slurry-like consistency with a resistivity of $6.5 \mathrm{ohm}-\mathrm{m}$. The sample was allowed to sit overnight for the third run. For the third run (figure 19) the water content was $17.6 \%$, and a resistivity of $7.6 \mathrm{ohm}-\mathrm{m}$.

For the first measurement, the resistivity and phase spectra are type A, and show a high-frequency dielectric dispersion and a broad, low-frequency dispersion at about $0.5 \mathrm{~Hz}$. The second and third run (figures 18 and 19) have type B spectra showing the high-frequency dispersion and a low-amplitude dispersion centered at about $100 \mathrm{~Hz}$. The PFE values range from 0.4 to $23.9 \%$, and decrease with the addition of water.

\section{$\underline{L V-102 \mathrm{P}}$}

This sample was collected from the goethite zone of the Venir site. XRD analysis indicates the sample contains major quartz; minor sanidine and muscovite; and trace goethite, jarosite and kaolinite. The sample was measured 3 different times. The first run (figure 20) had a water content of 3.7 weight percent, and a resistivity greater than 51,000 ohm-m. Water content was increased to $6.6 \%$ for the second run (figure 21), resulting in a resistivity of 6844 ohm-m. For the third run (figure 22) the water content was increased to $12.1 \%$, and the resistivity decreased to $1063 \mathrm{ohm}-\mathrm{m}$.

The phase spectra for the first and second measurements only have the high-frequency dispersion, and are neither type A or B spectra. The third measurement (figure 22) has a type A spectra and shows the resistivity to be strongly frequency dependent below $5.0 \mathrm{~Hz}$. The phase spectra shows a broad, 
high amplitude dispersion centered at $0.5 \mathrm{~Hz}$. PFE values range from 2.9 to $61.0 \%$ and increase with the addition of water.

\section{Discussion}

When water is added to a sample, the physical and chemical equilibrium of the sample is disturbed. Physically, the water must diffuse throughout the sample. As water hydrates the dry material, chemical reactions may occur that alter the electrical properties of the mine waste. Diffusion and some chemical reactions can be relatively slow, and it may take hours or days to reach physical and chemical equilibrium. Therefore, samples measured immediately after adding water may have different electrical properties than samples that are allowed to equilibrate for longer periods of time.

In the following discussions the condition of the samples may be referred to as "dry " or "wet". A "dry" sample measurement is the first measurement when the sample had the lowest water weight percent, typically less than $4 \%$. A "wet" sample measurement is a later run with a water weight percent of greater than about $9 \%$. Sample LV-1G2H is an exception; no "dry" measurement was completed.

The following discussion addresses the mineralogy of the samples. The mineralogy was determined using X-ray diffraction (XRD) methods (Table 1 ). It is important to note that the XRD method has a lower detection limit of about 3-5 percent by volume. Low concentrations of some minerals (e.g. $2 \%$ salt) may significantly effect the electrical properties of the sample. Also, the XRD method only detects crystalline material; amorphous (non-crystalline) material is not detected. Most of the Leadville samples contain a relatively large amount (20$40 \%$ ) of amorphous material. Therefore, the electrical properties of the mine waste may be effected by a mineral or chemical constituent not detected by the XRD analysis.

\section{Low-frequency Resistivity}

The resistivity of the Leadville samples varies from less than 5 to greater than 50,000 ohm-m, depending on water weight percent. For the "dry" measurements, less than $4 \%$ water, resistivities ranged from 164 to 51,000 ohm$\mathrm{m}$. For the "wet" measurements, resistivities ranged from 4.3 to $1062 \mathrm{ohm}-\mathrm{m}$.

As expected, the addition of water lowers the resistivity of most samples. However, the resistivity of sample LV-IG2H decreased from 6.1 to $5.5 \mathrm{ohm}-\mathrm{m}$ when the water percent decreased from 17.3 to $14.0 \%$, and sample LV-1WX decreased from 5.7 to $4.3 \mathrm{ohm}-\mathrm{m}$ when the water percent decreased from 15.6 to $9.7 \%$. This anomalous behavior is explained as follows. For their last measurement, the samples were returned to sample jars and mixed with any unused sample, then were allowed to sit overnight. During this relatively long, inter-measurement period, the solid mineral phase reacted with the added water. 
When measured the following day, the chemistry of the samples had changed to make them more conductive even though the water weight percent had decreased. In both cases, the final sample density increased, suggesting the overnight chemical change could be something as simple as dissolving soluble mineral phases to produce highly conductive pore water.

Resistivity and mineral composition.

Based on the mineralogy, the Leadville samples can be divided into two groups, jarosite and goethite. The two groups have significantly different water dependent electrical properties. Jarosite samples are those collected from "jarosite zones" as delineated by spectroscopy surveys (Swayze, 1997 and 2000). Jarosite samples contain minor amounts of jarosite and no goethite as determined by XRD analysis. Samples LV-IG2H, LV-1WX, LV-1A2B, and LV$2 \mathrm{QR}$ are the jarosite samples.

Goethite samples are those collected from "goethite zones" as delineated by spectroscopy surveys. The goethite zone identified by the spectroscopy survey may have goethite concentrations below the detectable limit of XRD analysis. Samples LV-2WX and LV-102P are goethite samples. XRD analysis only detected goethite in sample LV-102P. All the goethite samples contain jarosite as a trace amount.

The geothite and jarosite samples have significantly different lowfrequency resistivities. At low-water content (less than 4 weight percent), goethite samples are about an order of magnitude more resistive than jarosite samples. The two, low-water goethite measurements (3.7 and 3.8\%) had high resistivities $(51,233$ and $4895 \mathrm{ohm}-\mathrm{m})$, whereas the low-water jarosite measurements (3.4, 3.7 , and $3.7 \%$ ) had much lower resistivities $(164,1142$, and 429 ohm-m respectively).

At higher water content, greater than 9 weight percent, the wet goethite samples are typically two orders of magnitude more resistive than the jarosite samples. The "wet" goethite measurements, at 9.8 and $12.1 \%$ water, had resistivities of 659 and 1063 ohm-m, whereas wet jarosite measurements (14.0, $9.7,5.4$, and $17.6 \%$ water) had resistivities of $5.5,4.3,15.3$, and 7.6 ohm-m respectively.

The resistivity of the goethite samples shows a steady decrease with increasing water content (figure 23). At 19.2\%, sample LV-2WX4 was completely saturated, however, it's resistivity was still over $50 \mathrm{ohm}-\mathrm{m}$, nearly an order of magnitude more resistive than any of the saturated jarosite samples. The jarosite samples show a significantly different response to added water than do the goethite samples. A small increase in the amount of water drastically reduced the resistivity of the jarosite samples. Slightly above 5 weight percent water, the resistivity of all the jarosite samples dropped below $10 \mathrm{ohm}-\mathrm{m}$, then remains fairly constant being relatively unaffected by the addition of more water. 
The all the jarosite samples contain pyrite, either as a minor or trace component, whereas none of the goethite samples were observed to contain pyrite. The presence of pyrite may contribute to the conductivity of the sample in several ways. First, the mineral pyrite, an iron sulfide, is very conductive having an average resistivity of $0.3 \mathrm{ohm}-\mathrm{m}$ (Telford and others, 1976). In sufficient quantities, pyrite can lower the bulk resistivity of a sample due to its conductive nature. Secondly, the chemical weathering of pyrite produces $\mathrm{Fe}$ and $\mathrm{SO}_{4}$ ions and secondary mineral phases, such as copiapite, that can increase the conductivity of pore water. Copiapite, a soluble hydrous iron sulfate, was detected in trace amounts in three of the jarosite samples, but was not observed in sample LV-2QR or the goethite samples. With the addition of water, copiapite readily dissolves releasing iron and sulfate ions into the pore water which increases the conductivity of the sample. Dissolved copiapite also lowers the $\mathrm{pH}$ of the pore water.

It is interesting to note that when measured with a low water weight (< $4 \%$ ), sample LV-2QR had the highest resistivity of all the jarosite samples. Compositionally, this sample differs from the other jarosite samples in that it contains no copiapite, and only a trace of pyrite; whereas the other jarosite samples contain trace copiapite and minor pyrite. This observation, and the earlier observation that goethite samples do not contain either pyrite or copiapite, suggests the presence of pyrite and copiapite play a significant role in lowering a samples resistivity.

\section{Frequency dependency}

The Leadville samples exhibit frequency dependent electrical properties. The basic shape of the spectra vary as a function of water weight percent and as a function of mineralogy. For the jarosite samples, the dry measurement produced type A spectra. Once the water content of the jarosite samples was increased to $>5 \%$, the spectra changed to type B for all samples except LV1A2B. The goethite samples, wet or dry, generally have a type A spectra. One goethite sample, mixed to a slurry-like consistency with $19.2 \%$ water (figure 16), has neither an A or B type spectra, but does has a mid-frequency dispersion similar in frequency and amplitude to those observed in type B spectra.

Resistive samples, those greater than 100 ohm-m, typically have type A spectra. The low-frequency resistivity breaks associated with broad, moderateamplitude phase dispersions result from a combination of ionic conduction, cation exchange, and oxidation-reduction processes. The high-frequency resistivity breaks and associated high-amplitude phase dispersions are produced by dielectric polarization effects.

Conductive samples, those less than $10 \mathrm{ohm}-\mathrm{m}$, have type B spectra. The low-frequency resistivity breaks associated with broad, low-amplitude phase dispersions result from a combination of ionic conduction, cation exchange, and oxidation-reduction processes. The mid-frequency resistivity breaks associated 
with narrow, moderate-amplitude phase dispersions are ionic conduction effects related to pore-size. The high-frequency resistivity breaks and associated highamplitude phase dispersions are produced by dielectric polarization effects.

PFE values provide a quantitative measure of frequency dependency. For the jarosite samples, the PFE values decrease between the first and last measurement, whereas the goethite PFE values increase. Therefore, at low water weight percents $(<4 \%)$, the jarosite samples have high frequency dependency relative to the goethite samples. At high water weight percents $(>5 \%)$, the goethite samples have high PFE values relative to jarosite samples. For the samples that were allowed to sit overnight and equilibrate, the goethite samples had relatively high PFE values of 23.2 and $61.0 \%$, compared to 1.88 , $1.94,7.1$, and $0.7 \%$ for the jarosite samples.

\section{Linearity}

All of the Leadville samples exhibit some non-linear behavior at the lower frequencies. Cation exchange reactions associated with clay minerals and oxidation-reduction reactions involving sulfide minerals are relatively slow processes that are observed at low frequencies. In general, cation exchange reactions produce non-linear behavior observed as Hilbert distortion (HD) whereas oxidation-reduction reactions produce harmonic distortion (THD).

The Leadville samples contain several different clay minerals. Clays have high cation exchange capacities (CEC) relative to most non-clay minerals. All the samples contain muscovite and kaolinite. Two samples contain montmorillonite in trace and minor amounts respectively, and one sample contains illite. Of these clays, montmorillonite has the highest CEC, illite and muscovite have a moderate CEC, and kaolinite has the lowest.

Clay minerals tend to adsorb water. This adsorbed water and exchangeable cations makes the clay surface conductive. In relatively "dry" samples, cation exchange processes on the clay surface provides a conductive path for the electric current resulting in Hilbert distortion. In saturates claybearing material, the dominant conduction mechanism changes to ion migration through pore spaces and the HD decreases.

The dry jarosite samples had the highest HD values. Sample LV-1A2B had the highest HD of all the Leadville samples. The addition of water typically lowered the jarosite samples HD 25 to $50 \%$. However, water slightly increased the HD of sample LV-1A2B. The goethite samples, wet or dry, had relatively low $\mathrm{HD}$ values suggesting a lack of cation exchange processes.

Samples, LV-1A2B and LV-1G2H, contain montmorillonite in trace and minor amounts respectively. Sample LV-1G2H had the highest concentration of montmorillonite but does not show significant HD. However, sample LV-1G2H was not measured in a dry condition and conduction may be dominantly ionic 
through pore spaces. Sample LV-1A2B, has high HD at low-frequencies where cation exchange mechanisms are observed. The addition of water slightly increased the HD of this sample, suggesting clay remains a dominant conduction path in the saturated sample.

All of the jarosite samples contain minor or trace pyrite, where no pyrite was detected in the goethite samples. Oxidation-reduction reactions involving pyrite will produce non-linear behavior at low frequencies expressed as harmonic distortion. Generally, the jarosite samples have higher THD than the goethite samples but there are several exceptions.

Two jarosite samples, LV-1WX and LV-2QR, started with relatively high $T H D$, then show a significant decrease for the last measurement suggesting a decrease in oxidation-reduction processes as the water content was increased. When these samples were dry, the conductive sulfide minerals are a significant mode of conduction and polarization. Oxidation-reduction processes associated with the sulfide minerals, produced the relatively high THD. As the water content increases the dominant mode of conduction shifts to ionic conduction through pore water, the role of the sulfide minerals in charge transport diminished, and the THD decreased.

Sample LV-1G2H (figures 2-5) has two distinct THD peaks at different frequencies. The high-frequency peak, at about $10 \mathrm{~Hz}$, has a relatively low amplitude compared to the low-frequency peak. The two peaks suggest the sulfide minerals occur in two different modes, probably related to grain-size. Electric charges build up faster on small grains due to surface area effects. Therefore, polarization effects due to small mineral grains are seen a high frequencies.

Sample LV-1A2B had the highest overall THD values, starting at 2.2 and increasing to $8.8 \%$ for the final measurement. The THD increase suggests an increase in oxidation-reduction activity. Interestingly, sample LV-1A2B was the only jarosite sample not to have a conductive type B spectra. The lack of a type $B$ spectra, the high THD, and the high Hilbert distortion suggests both oxidationreduction and cation exchange are dominant mechanisms in sample LV-1A2B.

\section{Summary}

The Leadville samples exhibit frequency dependent electrical properties that vary as a function of water content and mineralogy. The main factor controlling the electrical properties was water content. The resistivity of the samples decreased as the water content increased. Relatively dry samples, with less than $4 \%$ water by weight, had resistivities ranging from 164 to $51,233 \mathrm{ohm}-$ $\mathrm{m}$. Relatively wet samples, with greater than $9 \%$ water, had resistivities ranging from 4.3 to $1063 \mathrm{ohm}-\mathrm{m}$. Dry jarosite samples are typically and order of magnitude more conductive than dry goethite samples. This is attributed to the 
presence of pyrite, and conductive weathering by products, in the jarosite samples. Two of the jarosite samples also contain montmorillonite, a conductive clay mineral.

With the addition of water, the resistivity of the jarosite samples typically decreased by two orders of magnitude, where as the goethite samples decreased about one order of magnitude. Jarosite samples show a rapid resistivity decrease relative to goethite samples. The large, rapid resistivity decrease observed in the jarosite samples is attributed to soluble mineral phases (i.e. copiapite) dissolving, which increases ionic conduction through pore fluids.

The frequency dependency also varies as a function of water content and mineralogy. Two characteristic spectral shapes were observed for most of the measurements. Type A spectra are observed in resistive, dry jarosite and goethite samples. The moderate amplitude, low-frequency dispersion that characterizes type A spectra results from a combination of ionic conduction, cation exchange, and oxidation-reduction processes. Type B spectra are observed in conductive, wet jarosite samples. The moderate amplitude, midfrequency dispersion that characterizes type B spectra results from ionic conduction effects related to pore-size.

Based on the PFE data, dry jarosite samples are more frequency dependent that dry goethite samples. The higher PFE is attributed to polarization mechanisms associated with pyrite and clay minerals present in the jarosite samples. Wet jarosite samples are typically less frequency dependent that wet goethite samples. The lower PFE is attributed to ionic conduction through conductive pore water in the jarosite samples.

The Leadville samples also exhibit non-linear electrical properties that vary as a function of water content and mineralogy. Dry jarosite samples have the highest Hilbert distortion (HD) values, whereas goethite samples, wet or dry, have relatively low HD values. The HD observed in the jarosite samples is attributed to the presence of clay minerals and associated cation exchange reactions. Water typically lowers the HD of jarosite samples 25 to $50 \%$. The total harmonic distortion (THD) data does not show a strong correlation with mineralogy or water content, however, jarosite samples generally have higher THD than goethite samples. The THD observed in the jarosite samples is attributed to oxidation-reduction reactions involving pyrite.

\section{Acknowledgements}

The author would like to thank Dr. Gary Olhoeft, Colorado School of Mines, for access to the NLCR system. Dan Jones is acknowledged for his assistance in making the NLCR measurements. Gregg Swayze and Steve Sutley, USGS, are acknowledged for sample collection and XRD analysis. 


\section{References}

Campbell, D., and Horton, R.J., 2000, Graphs and tables used to describe electrical measurements of samples of unconsolidated material, USGS Petrophysical Laboratory-Denver: U.S. Geological Survey Open-File Report 000377, $16 \mathrm{p}$.

Jones, D.P., 1997, Investigation of clay-organic reactions using complex resistivity: Unpublished Masters thesis, Colorado School of Mines, $378 \mathrm{p}$.

Olhoeft, G.R., 1979, Nonlinear electrical properties, in Nonlinear behavior of molecules, atoms and ions in electric, magnetic or electromagnetic fields: Neel, L., Ed., Elsevier Science Publishing Co., pp. 395-410.

Olhoeft, G.R., 1985, Low-frequency electrical properties: Geophysics, v. 50, no. 12, pp. 2492-2503.

Swayze, G.A., Smith K.S., Clark, R.N., Sutley, S.J., Pearson, R.M., Vance, J.S., Hageman, P.L., Briggs, P.H., Meier, A.L., Singleton, M.J., and Roth, S., 2000, Using imaging spectroscopy to map acidic mine waste: Environmental Science and Technology, v. 34, p. 47-54.

Telford, W.M., Geldart, L.P., Sheriff, R.E., and Keys, D.A., 1976, Applied Geophysics: Cambridge University Press, New York, NY, 860 p. 
Table 1. Mineral analysis of the Leadville samples obtained by X-ray diffraction and spectroscopic analysis. Shaded columns indicate the goethite bearing samples.

\begin{tabular}{|l|c|c|c|c|c|c|}
\hline Mineral & LV-WX1 & $\mathbf{L V - 1 A 2 B}$ & $\mathbf{L V - 1 G 2 H}$ & $\mathbf{L V - 2 Q R}$ & $\mathbf{L V - 2 W X}$ & $\mathbf{L V - 1 0 2 P}$ \\
\hline Quartz & major & major & minor & major & major & major \\
\hline Muscovite & minor & minor & minor & minor & minor & minor \\
\hline Jarosite & minor & minor & minor & minor & trace & trace \\
\hline Kaolinite & trace & trace & trace & trace & trace & trace \\
\hline Feldspar & trace & trace & minor & NO & trace & minor \\
\hline Pyrite & minor & minor & minor & trace & NO & NO \\
\hline Montmorillonite & NO & trace & minor & NO & NO & NO \\
\hline Copiapite & trace & trace & trace & NO & NO & NO \\
\hline Illite & NO & NO & NO & minor & NO & NO \\
\hline Goethite & NO & NO & NO & NO & trace & trace \\
\hline
\end{tabular}

$\mathrm{NO}=$ Not Observed

Table 2. Low-frequency resistivity measurement, water weight percent, and density for the Leadville samples. The low-frequency resistivity measurements were typically made at a frequency of $0.002 \mathrm{~Hz}$. Shaded rows indicate goethite bearing samples.

\begin{tabular}{|c|c|c|c|}
\hline Sample/run & Resistivity (ohm-m) & Percent water & Density $\left(\mathrm{g} / \mathrm{cm}^{3}\right)$ \\
\hline LV-IG2H & 6.3 & 17.3 & 1.66 \\
\hline LV-IG2H2 & 6.1 & 17.3 & 1.66 \\
\hline LV-IG2H3 & 6.1 & 17.3 & 1.66 \\
\hline LV-IG2H4 & 5.5 & 14.0 & 2.02 \\
\hline LV-1WX & 429.1 & 3.7 & 1.39 \\
\hline LV-1WX2 & 421.6 & 3.7 & 1.39 \\
\hline LV-1WX3 & 5.7 & 15.6 & 1.59 \\
\hline LV-1WX4 & 4.6 & 9.7 & 2.04 \\
\hline LV-1WX5 & 4.3 & 9.7 & 2.04 \\
\hline LV-1A2B & 164.2 & 3.4 & 1.64 \\
\hline LV-1A2B5 & 15.3 & 5.4 & 1.79 \\
\hline LV-2WX1 & 4895 & 3.8 & 1.59 \\
\hline LV-2WX2 & 165.7 & 11.7 & 1.73 \\
\hline LV-2WX3 & 658.8 & 9.8 & 1.66 \\
\hline LV-2WX4 & 51.9 & 19.2 & 2.12 \\
\hline LV-2QR1 & 1142.3 & 3.7 & 1.45 \\
\hline LV-2QR2 & 6.5 & 22.5 & 1.79 \\
\hline LV-2QR3 & 7.6 & 17.6 & 1.92 \\
\hline LV-102P1 & 51233 & 3.7 & 1.54 \\
\hline LV-102P2 & 6843.7 & 6.6 & 1.59 \\
\hline LV-102P3 & 1062.8 & 12.1 & 1.65 \\
\hline
\end{tabular}

“*” indicates measurement made at $0.02 \mathrm{~Hz}$. 
Table 3. Percent frequency effect (PFE) values calculated from $0.1 \mathrm{~Hz}$ and $1.0 \mathrm{~Hz}$ data. Shaded rows indicate goethite bearing samples.

\begin{tabular}{|c|c|}
\hline Sample/run & PFE \\
\hline LV-IG2H & 2.2 \\
\hline LV-IG2H2 & 1.9 \\
\hline LV-IG2H3 & 1.81 \\
\hline LV-IG2H4 & 1.88 \\
\hline LV-1WX & 21.1 \\
\hline LV-1WX2 & 19.6 \\
\hline LV-1WX3 & 2.14 \\
\hline LV-1WX4 & 2.14 \\
\hline LV-1WX5 & 1.94 \\
\hline LV-1A2B & 12.6 \\
\hline LV-1A2B5 & 7.1 \\
\hline LV-2WX1 & 6.3 \\
\hline LV-2WX2 & 2.1 \\
\hline LV-2WX3 & 32.3 \\
\hline LV-2WX4 & 0.11 \\
\hline LV-2QR & 23.9 \\
\hline LV-2QR2 & 0.4 \\
\hline LV-2QR3 & 0.7 \\
\hline LV-102P1 & 2.9 \\
\hline LV-102P2 & 7.1 \\
\hline LV-102P3 & 61.0 \\
\hline
\end{tabular}




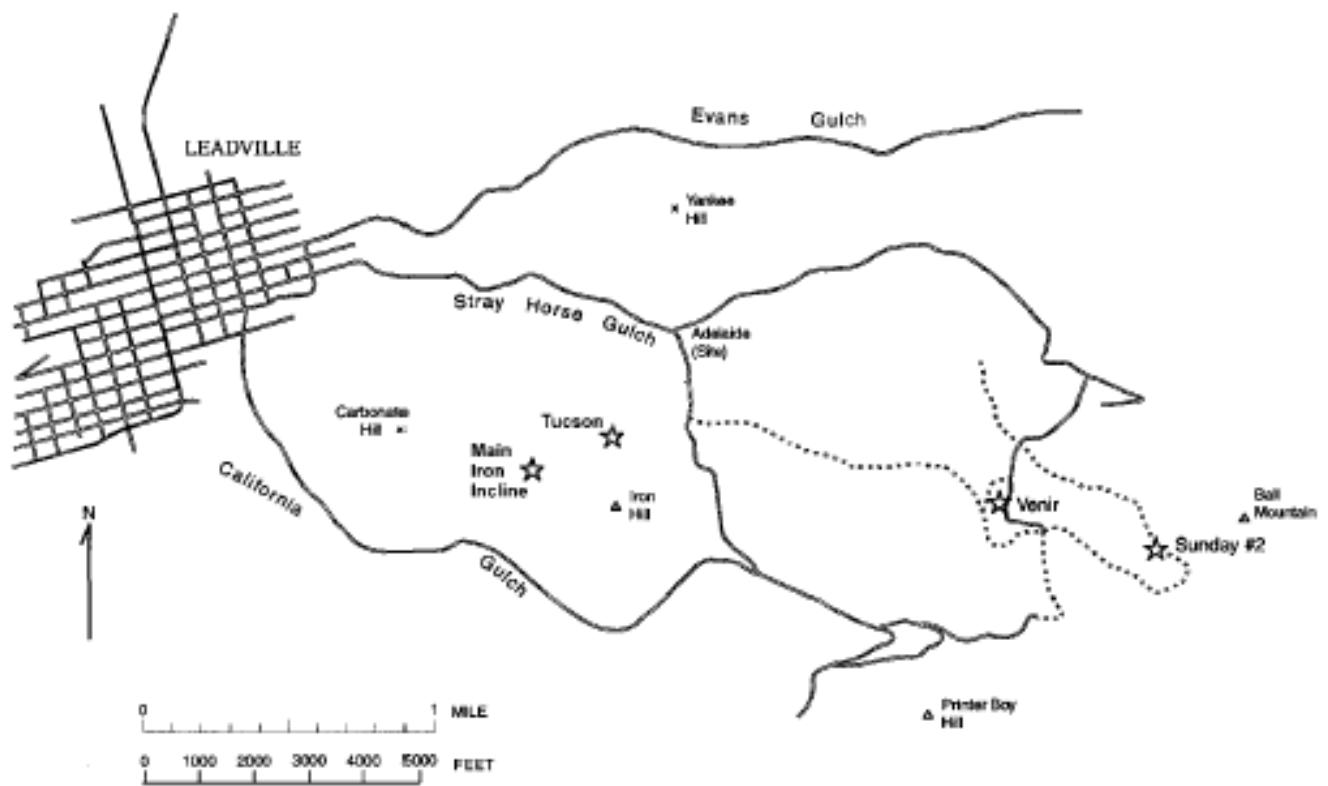

Figure1. Map of the Leadville Mining District showing the location of the Sunday \#2 and Venir mine sites.

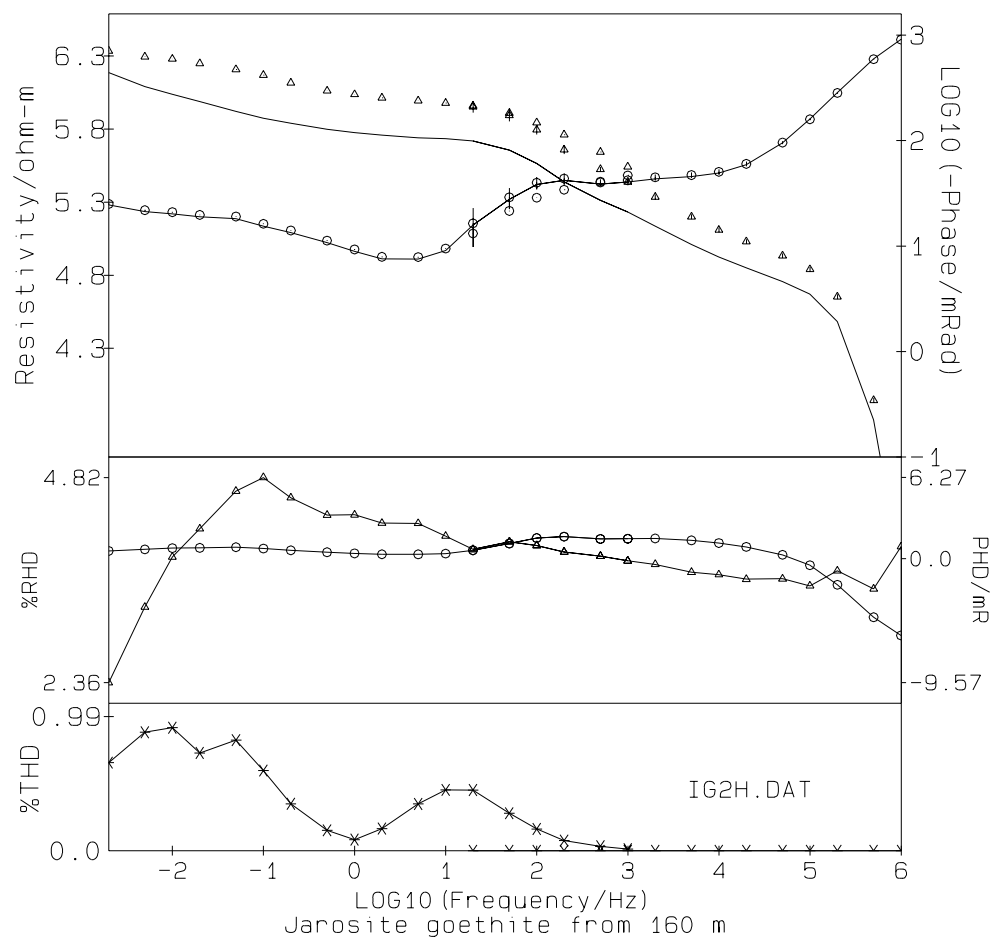

Figure 2. Sample LV-1G2H. First measurement, $17.3 \%$ water. 


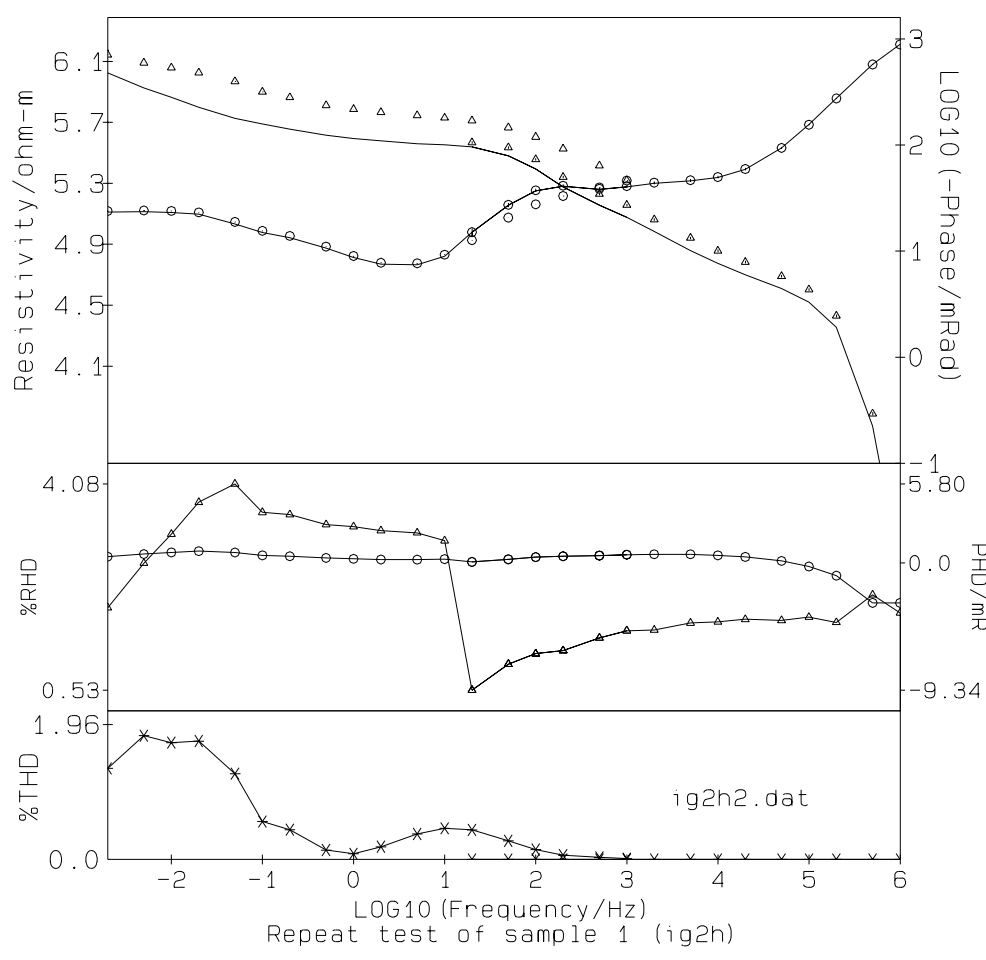

Figure 3 Sample LV-1G2H. Second measurement, repeat made 30 minutes after the first measurement. $17.3 \%$ water.

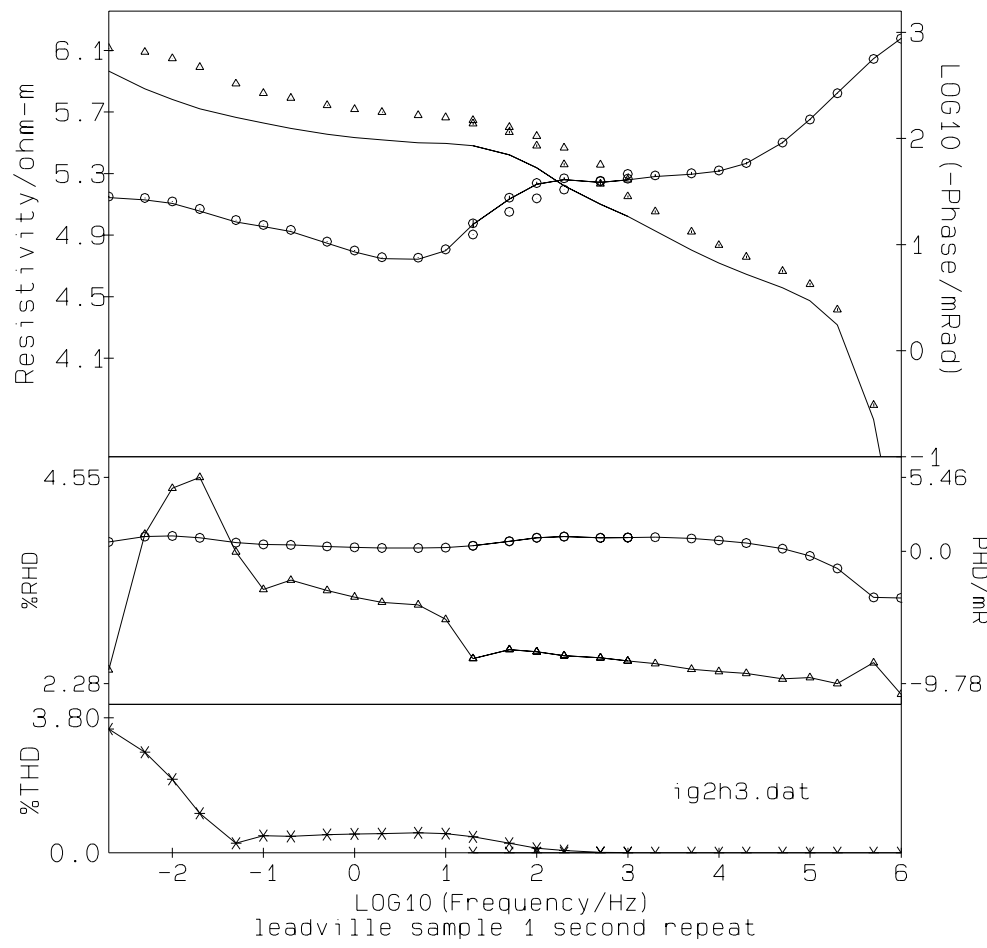

Figure 4 Sample LV-1G2H. Third measurement, repeat made 60 minutes after the first measurement. $17.3 \%$ water. 


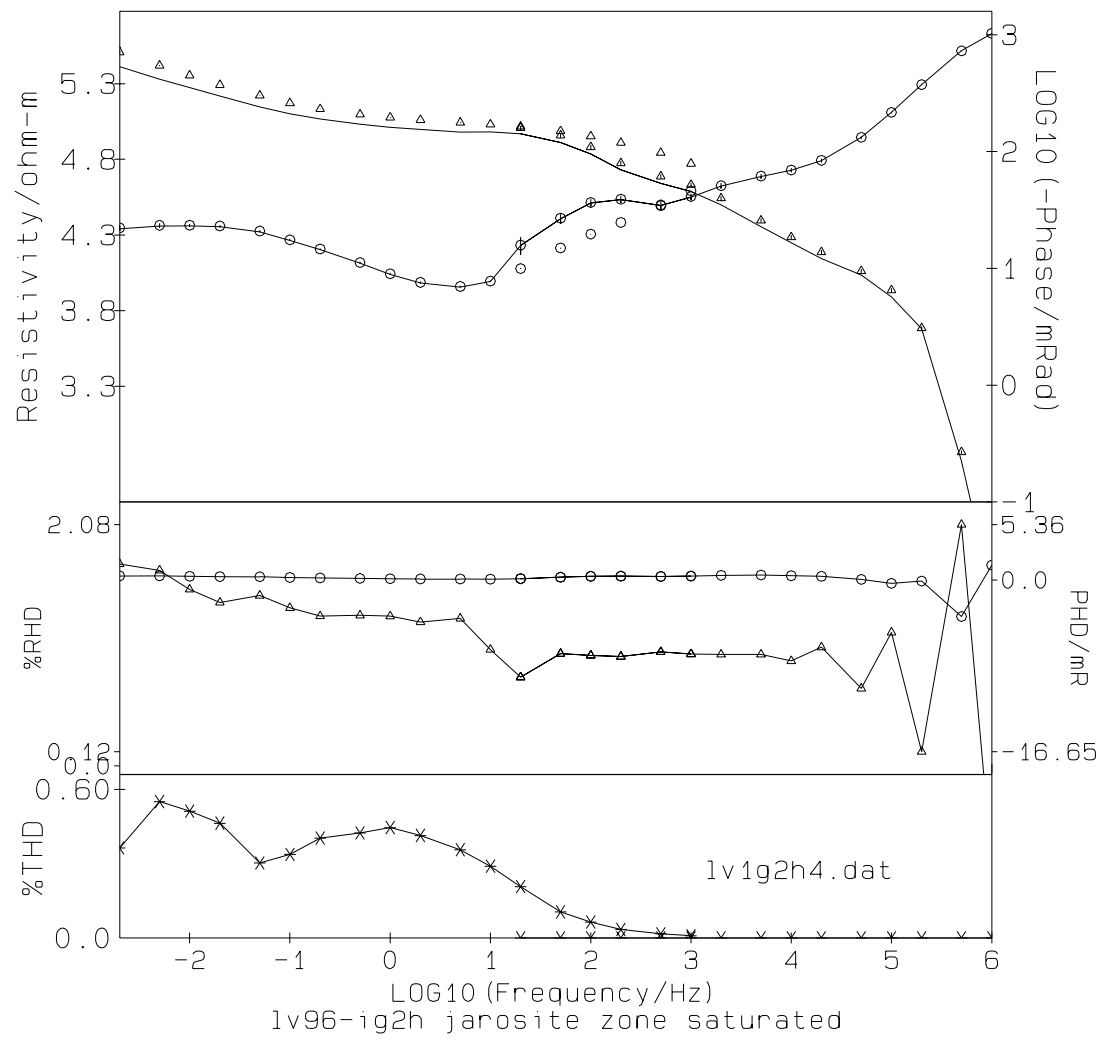

Figure 5 Sample LV-1G2H. Fourth measurement, sample allowed to sit overnight, $14.0 \%$ water.

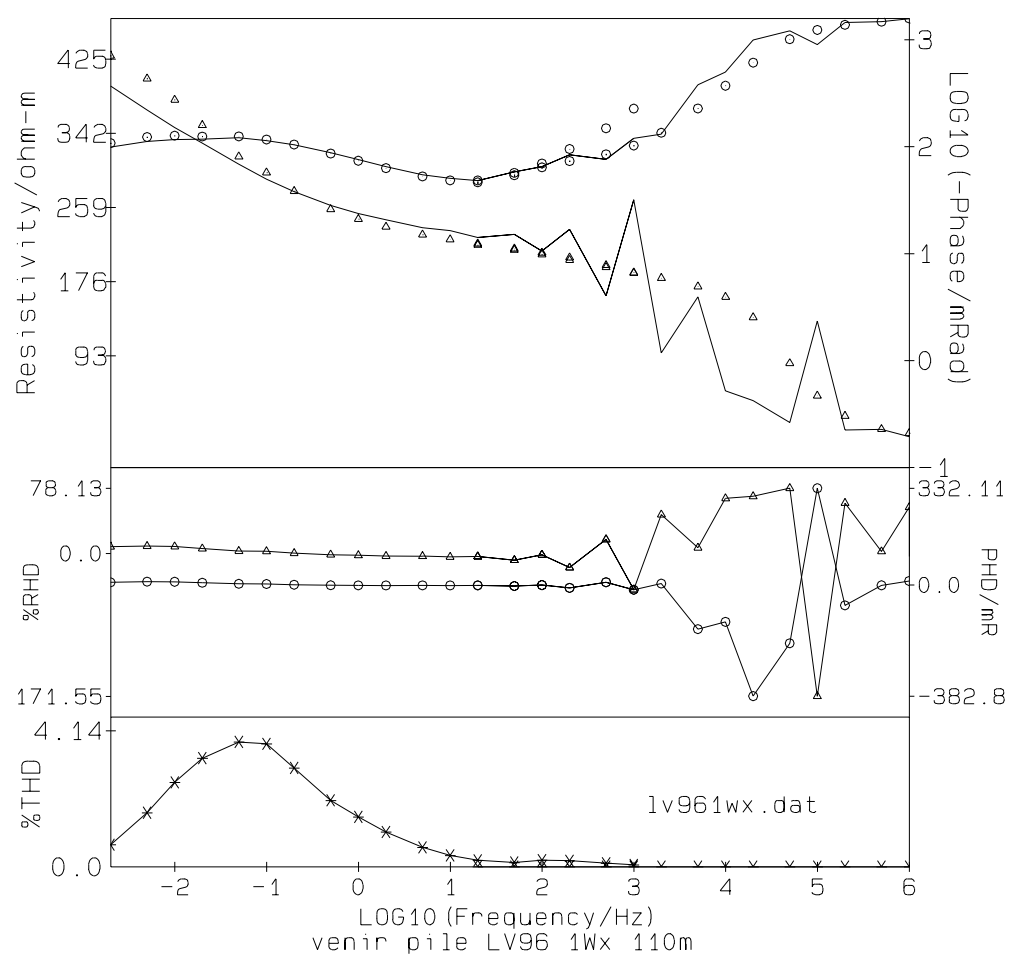

Figure 6. Sample LV-1WX. First measurement, 3.7\% water. 


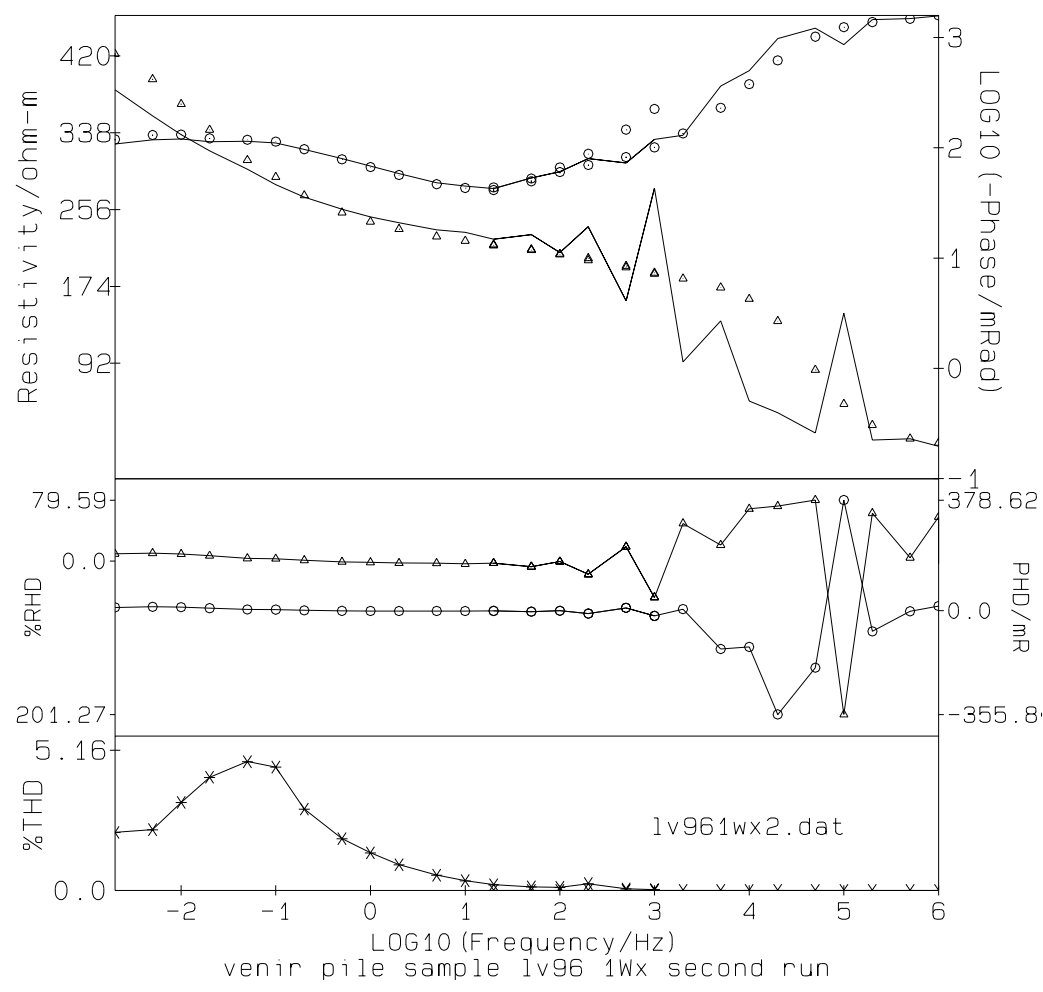

Figure 7. Sample LV-1WX. Second measurement, repeat measurement made 30 minutes after first, $3.7 \%$ water.

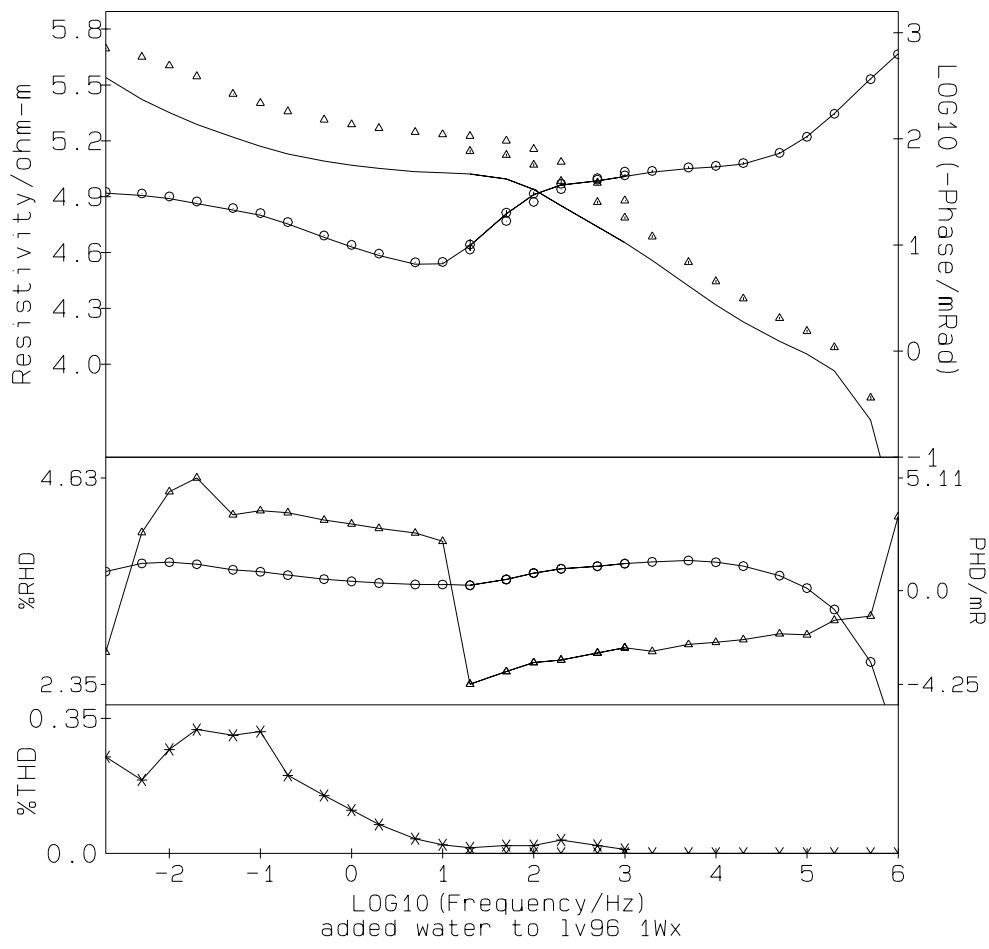

Figure 8. Sample LV-1WX. Third measurement, water increased to $15.6 \%$. 


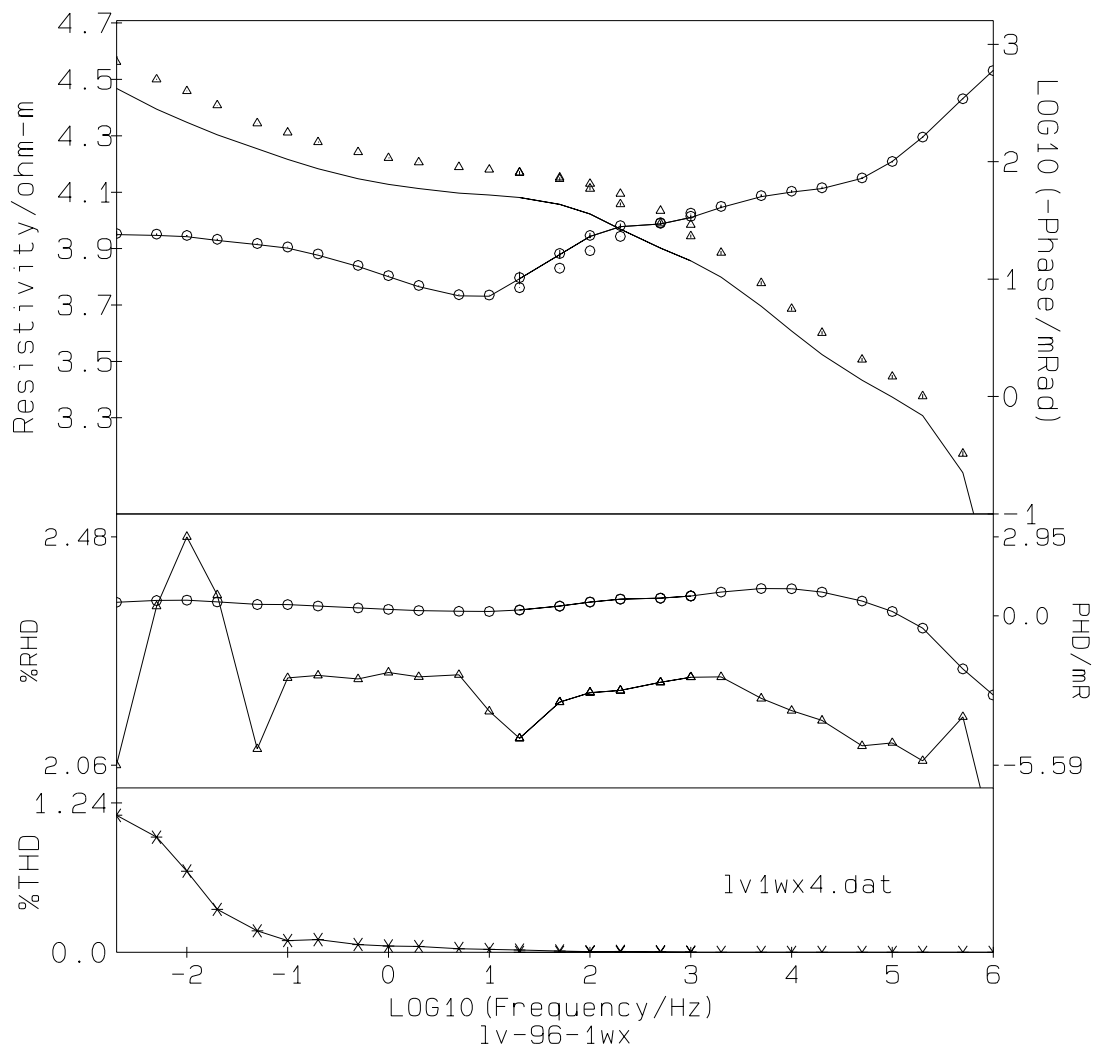

Figure 9. Sample LV-1WX. Fourth measurement, sample was allowed to sit overnight, water content $9.7 \%$

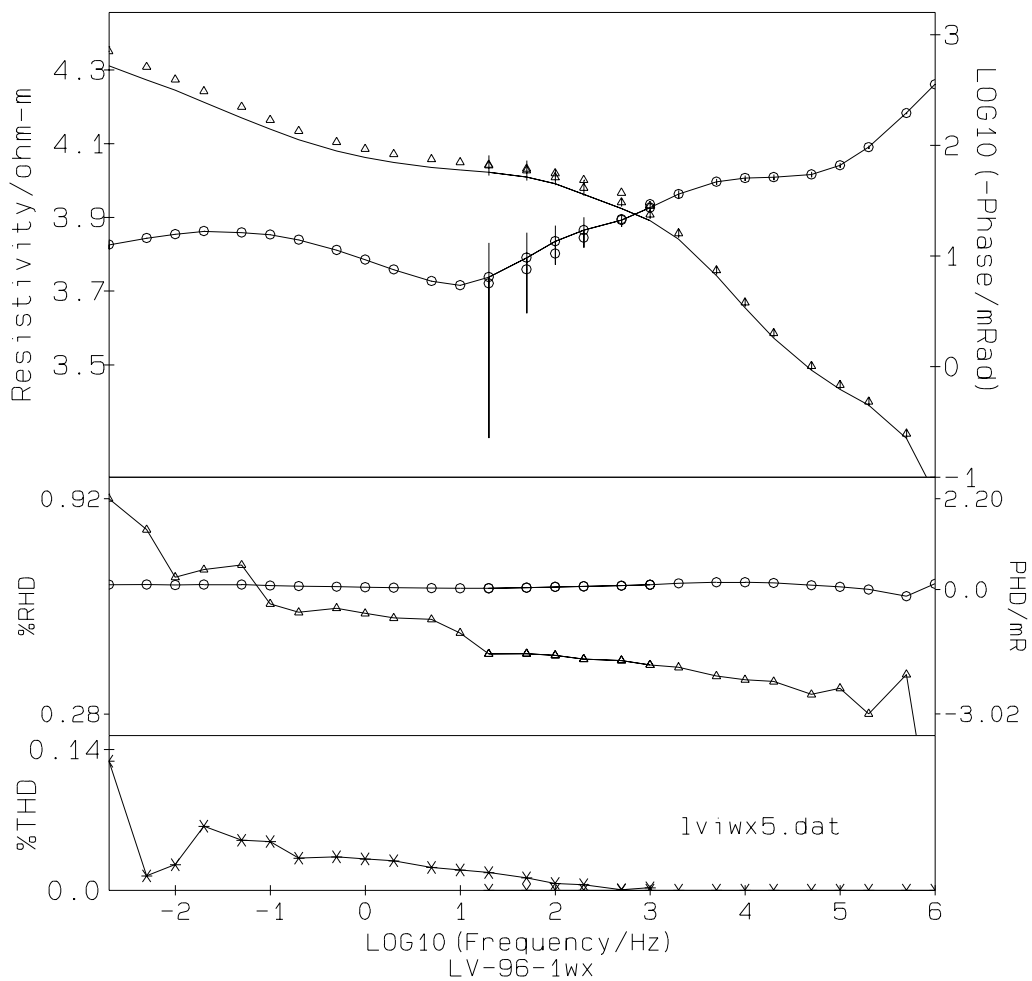

Figure 10. Sample LV-1WX. Fifth measurement, repeat using a smaller volume sample holder, water content $9.7 \%$ 


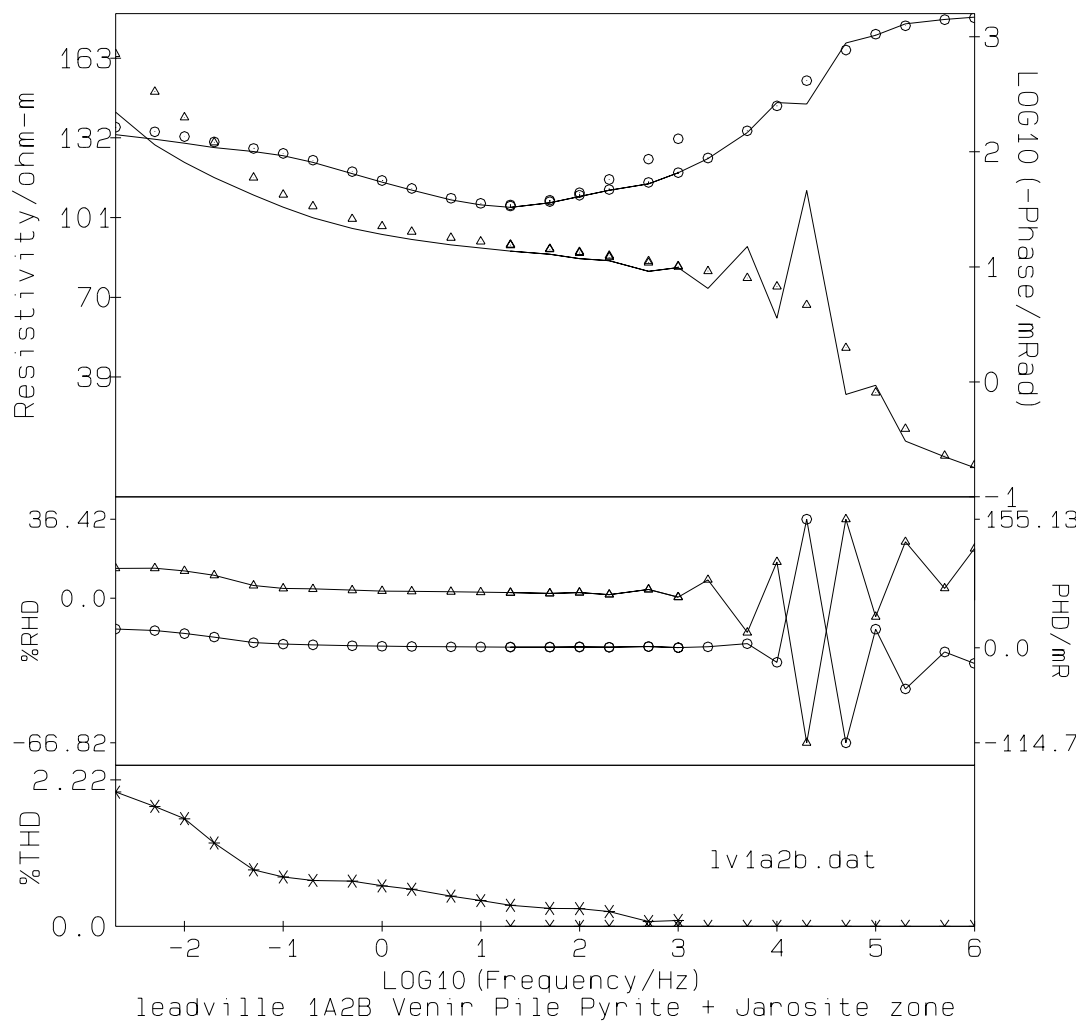

Figure 11. Sample LV-1A2B. First measurement, 3.4\% water.

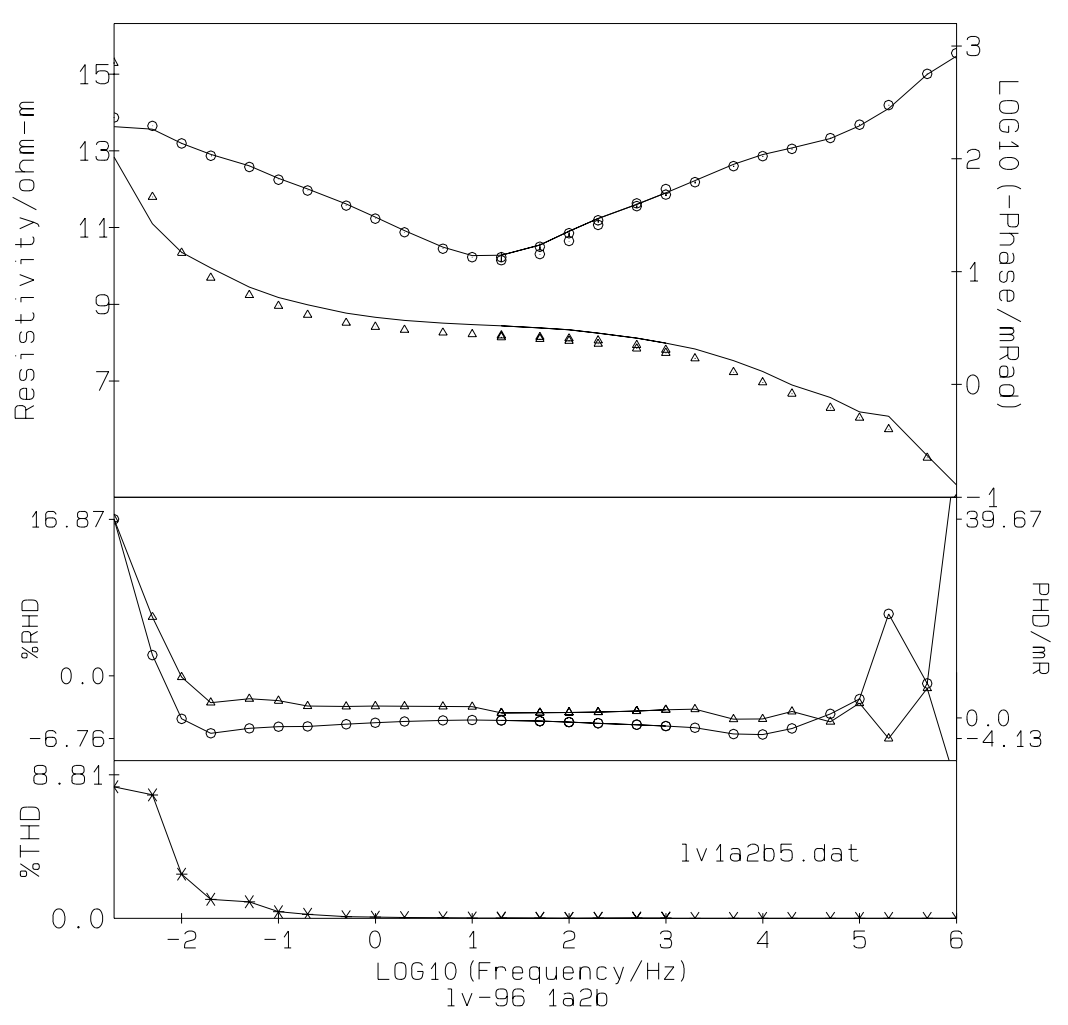

Figure 12. Sample LV-1A2B, Second measurement, sample was allowed to sit overnight, water content $5.4 \%$. 


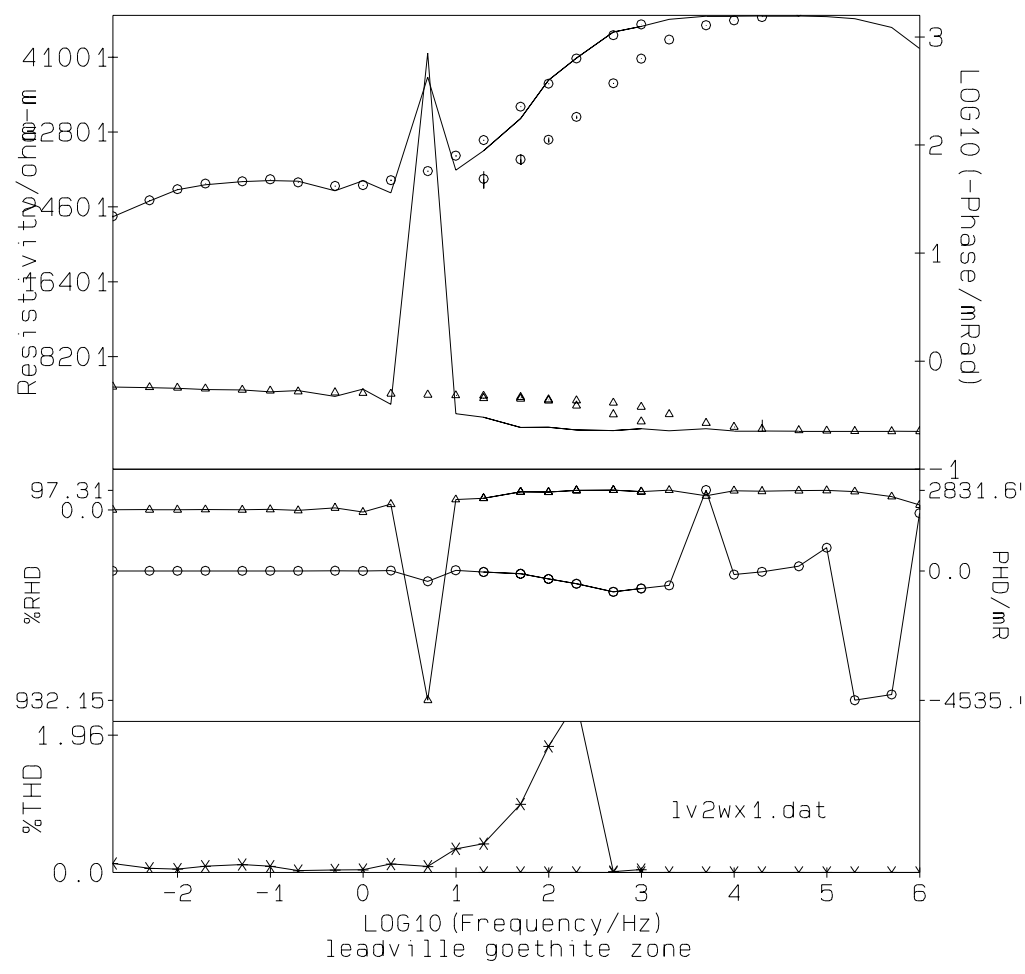

Figure 13. Sample LV-2WX. First measurement, water content 3.8\%.

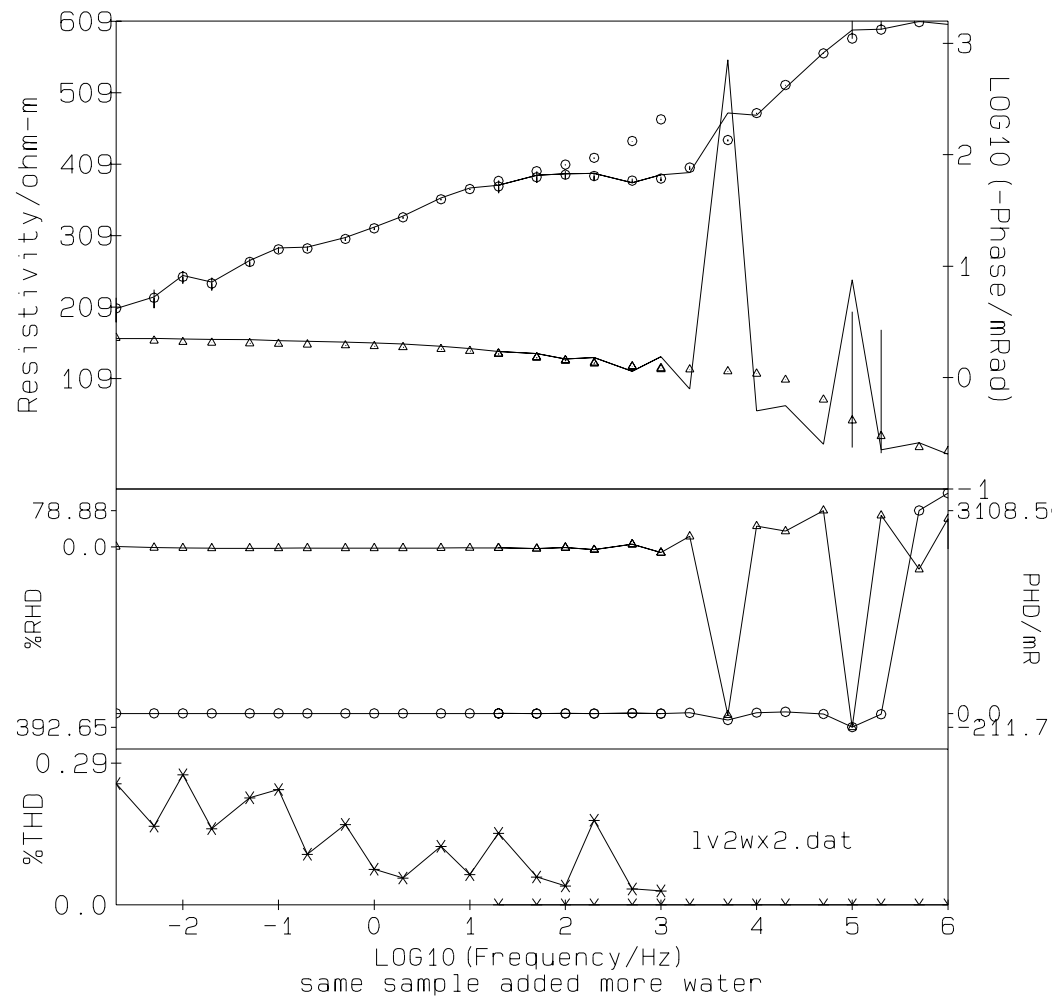

Figure 14. Sample LV-2WX. Second measurement, water content increased to $11.7 \%$. 


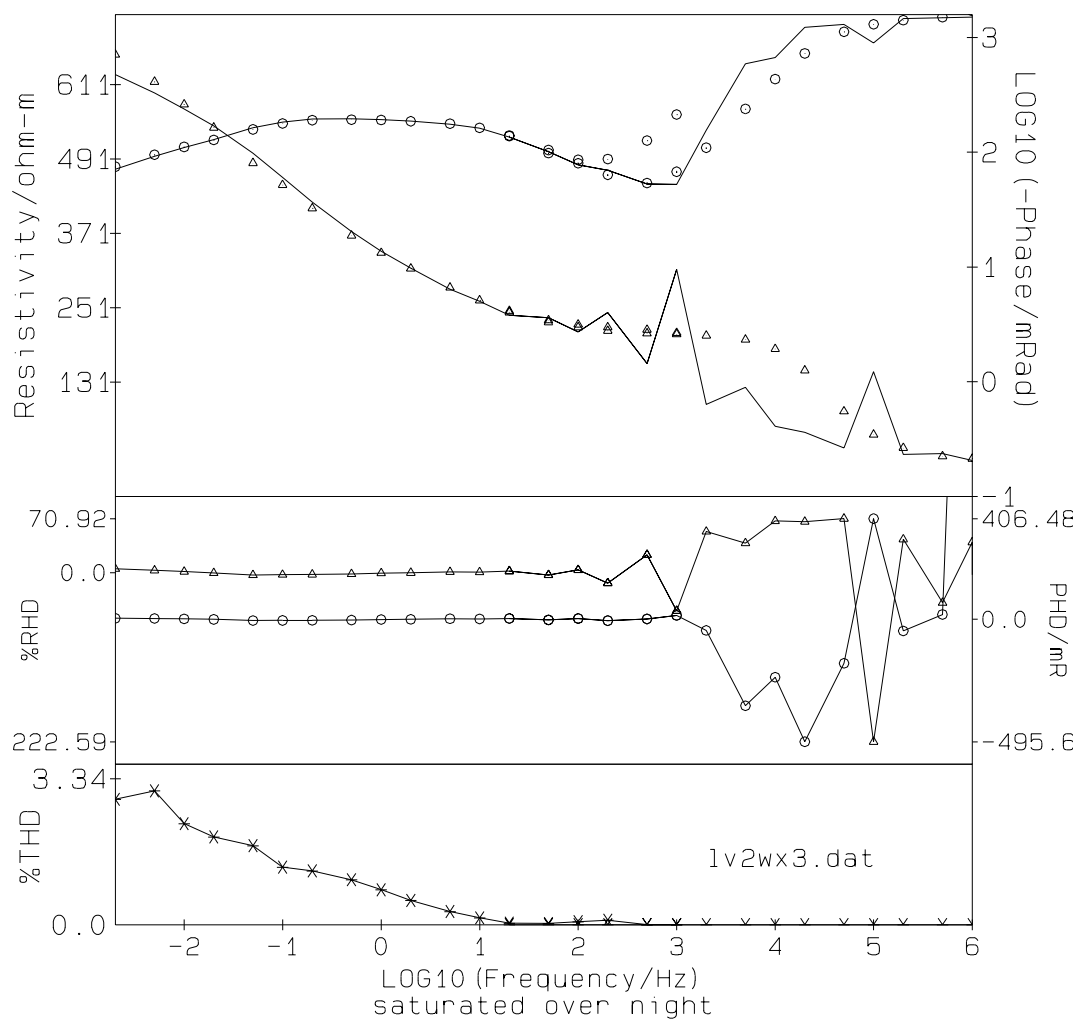

Figure 15. Sample LV-2WX. Third measurement, sample was allowed to sit overnight, water content $9.8 \%$.

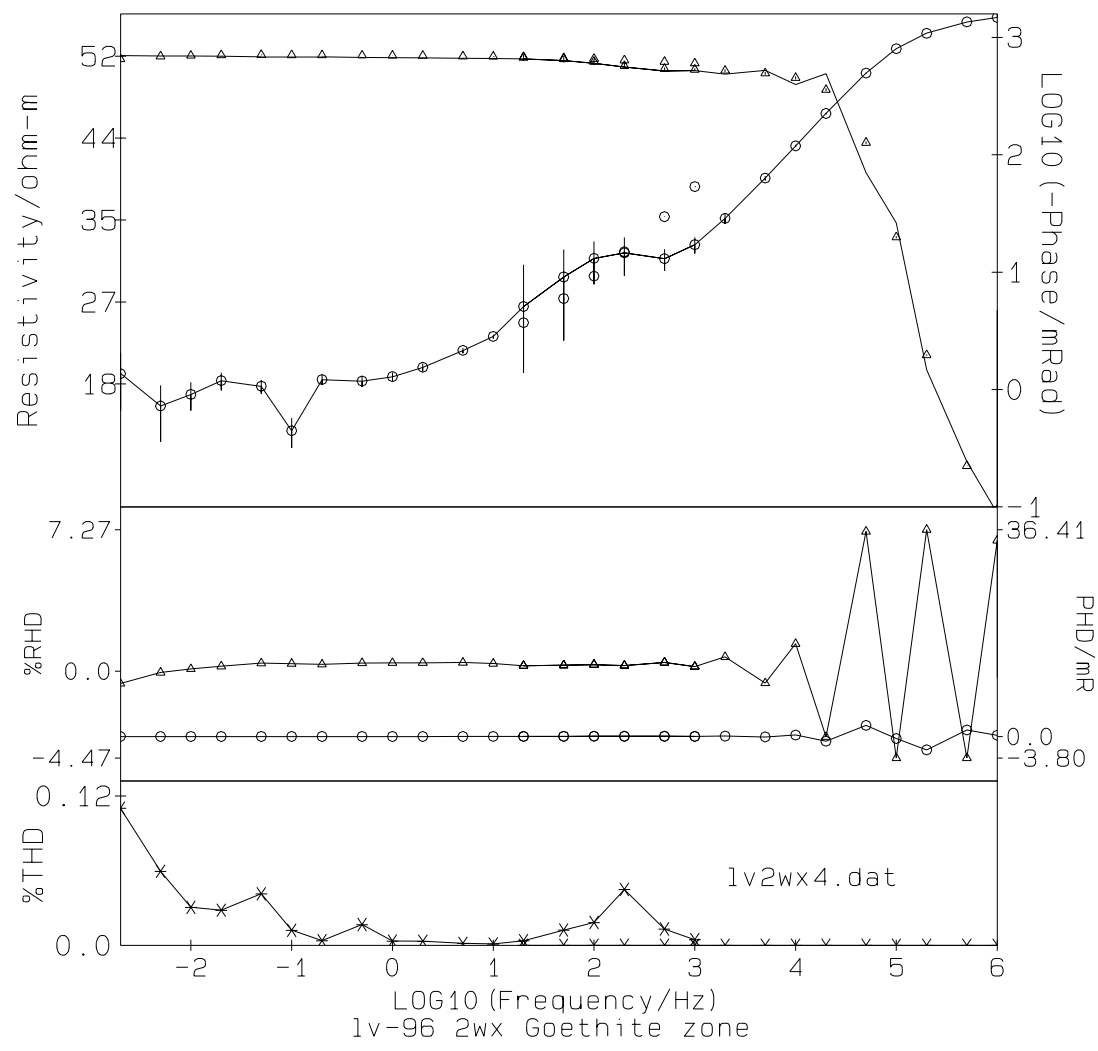

Figure 16. Sample LV-2WX. Fourth measurement, water content increased to $19.2 \%$, resulting in a slurry-like consistency. 


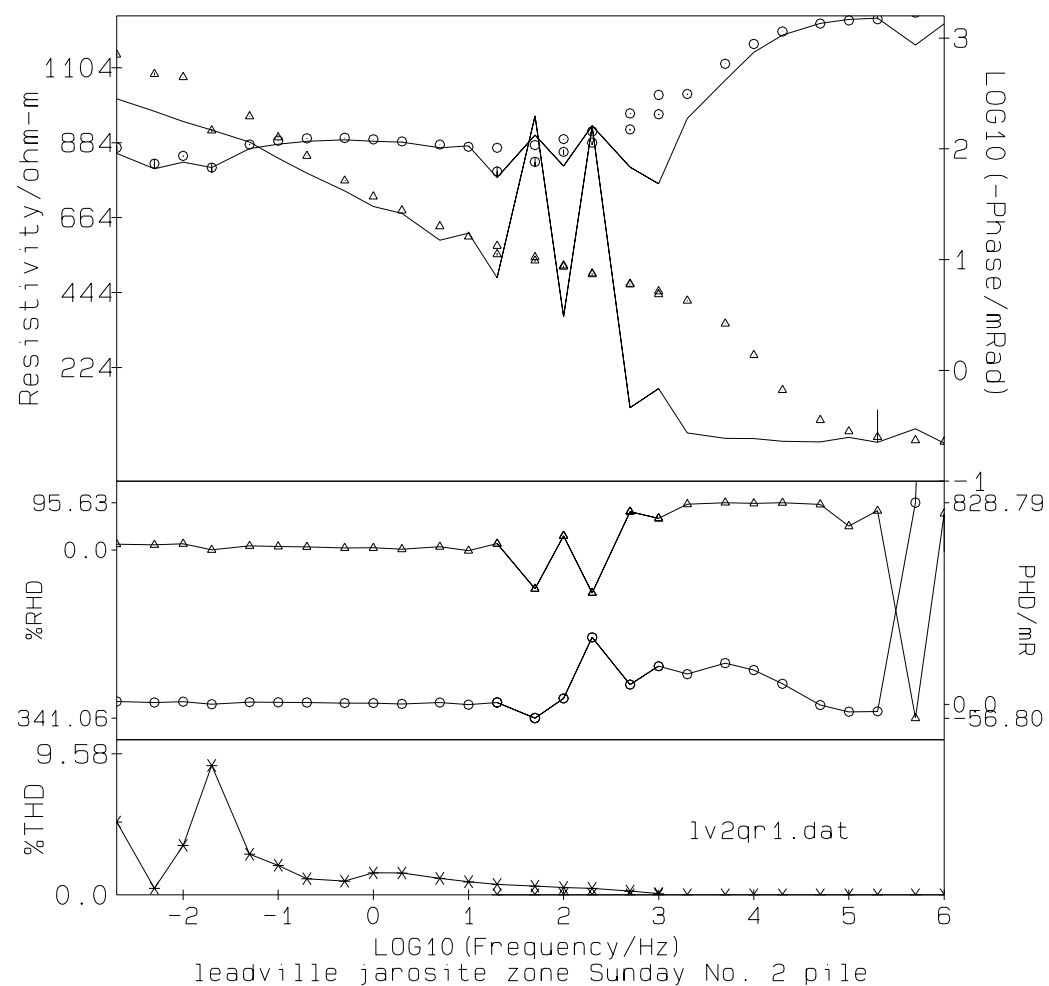

Figure 17. Sample LV-2QR. First measurement, water content 3.7\%.

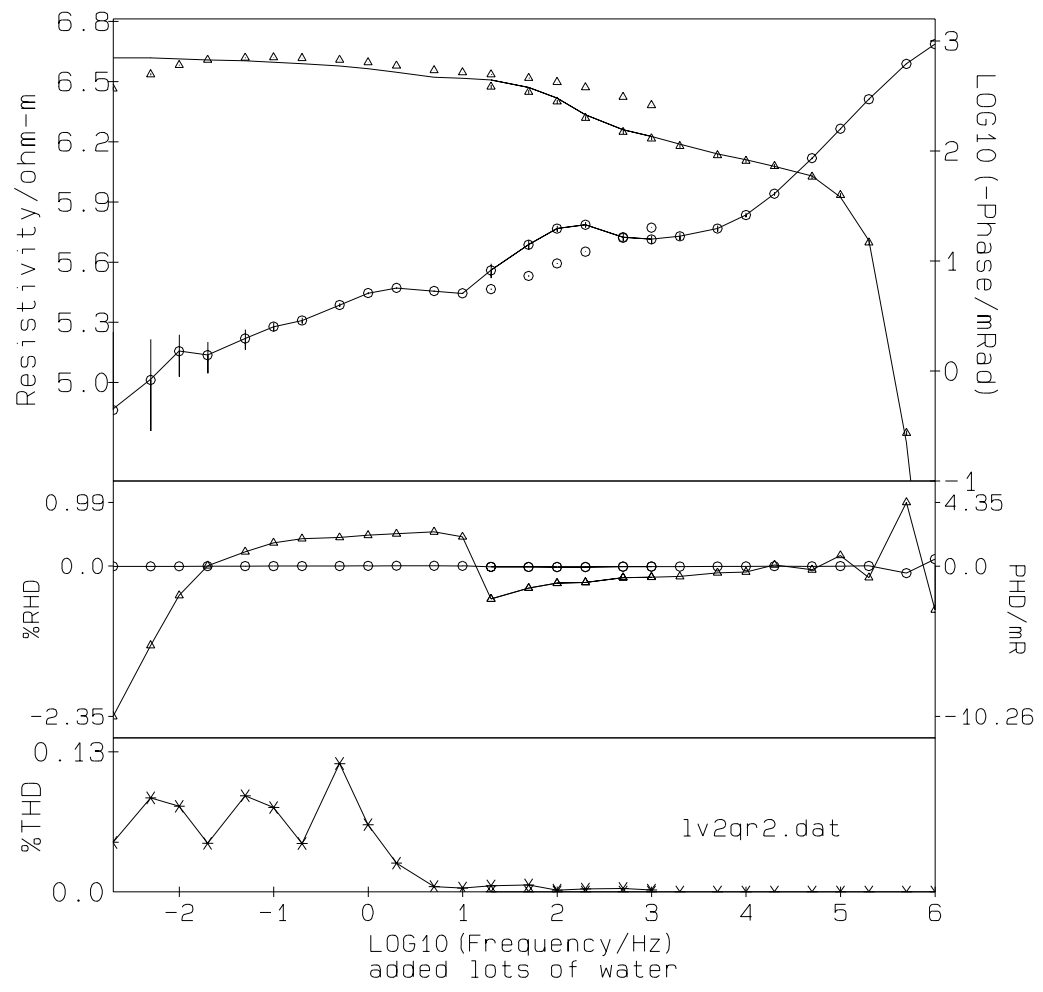

Figure 18. Sample LV-2QR. Second measurement, water content was increased to $22.5 \%$, resulting in a slurry-like consistency. 


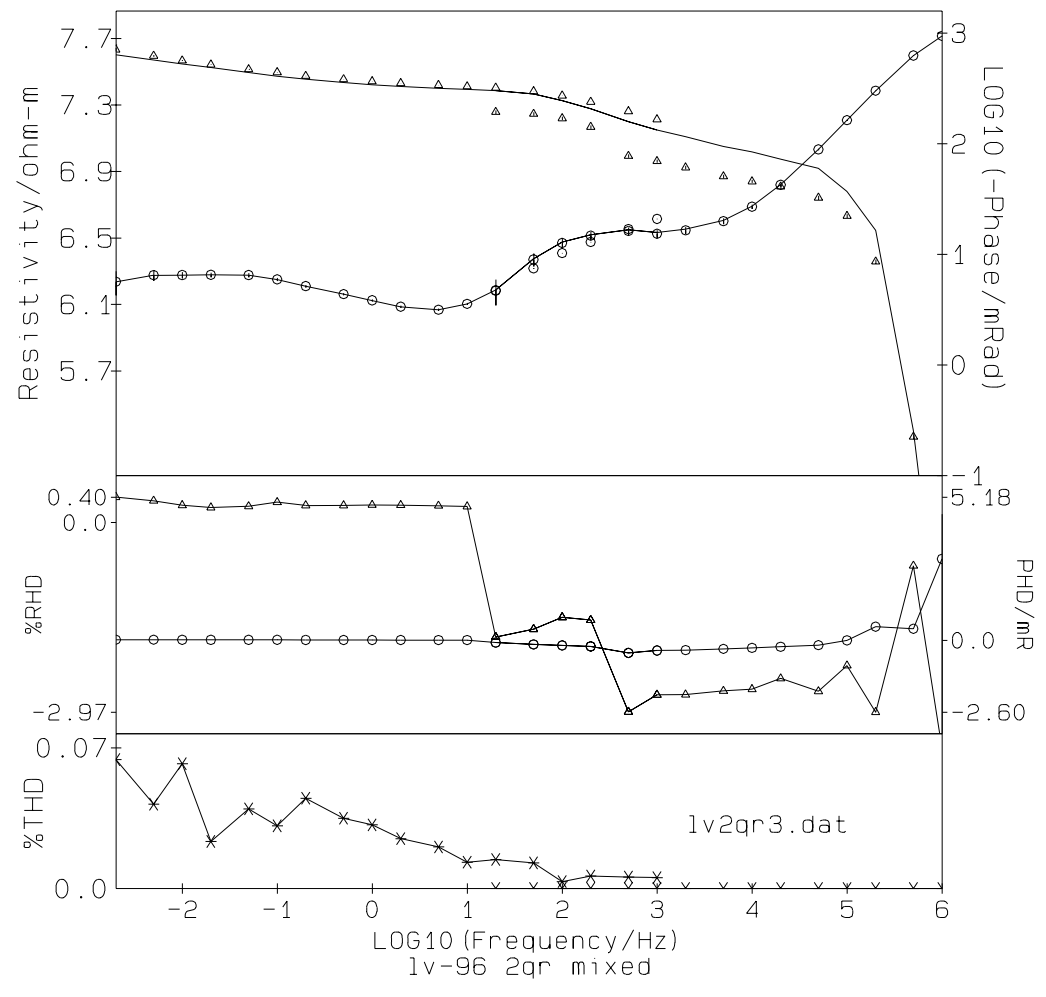

Figure 19. Sample LV-2QR. Third measurement, sample was allowed to sit overnight, water content was $17.6 \%$.

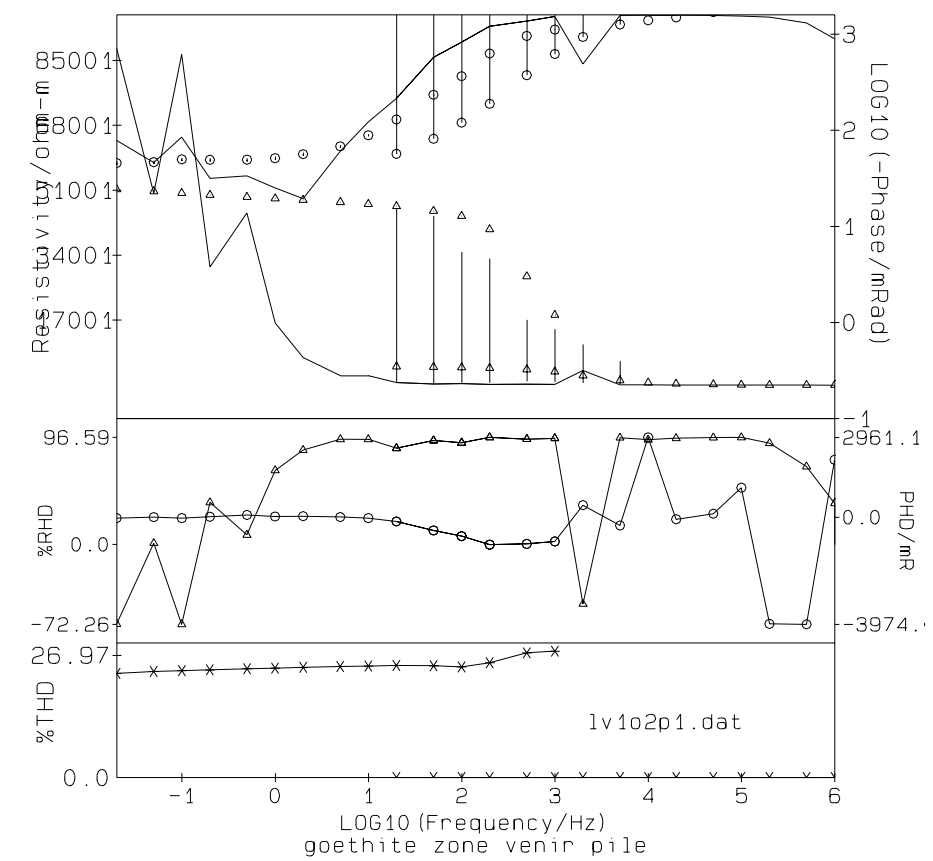

Figure 20. Sample LV-102P. First measurement, water content $3.7 \%$. 


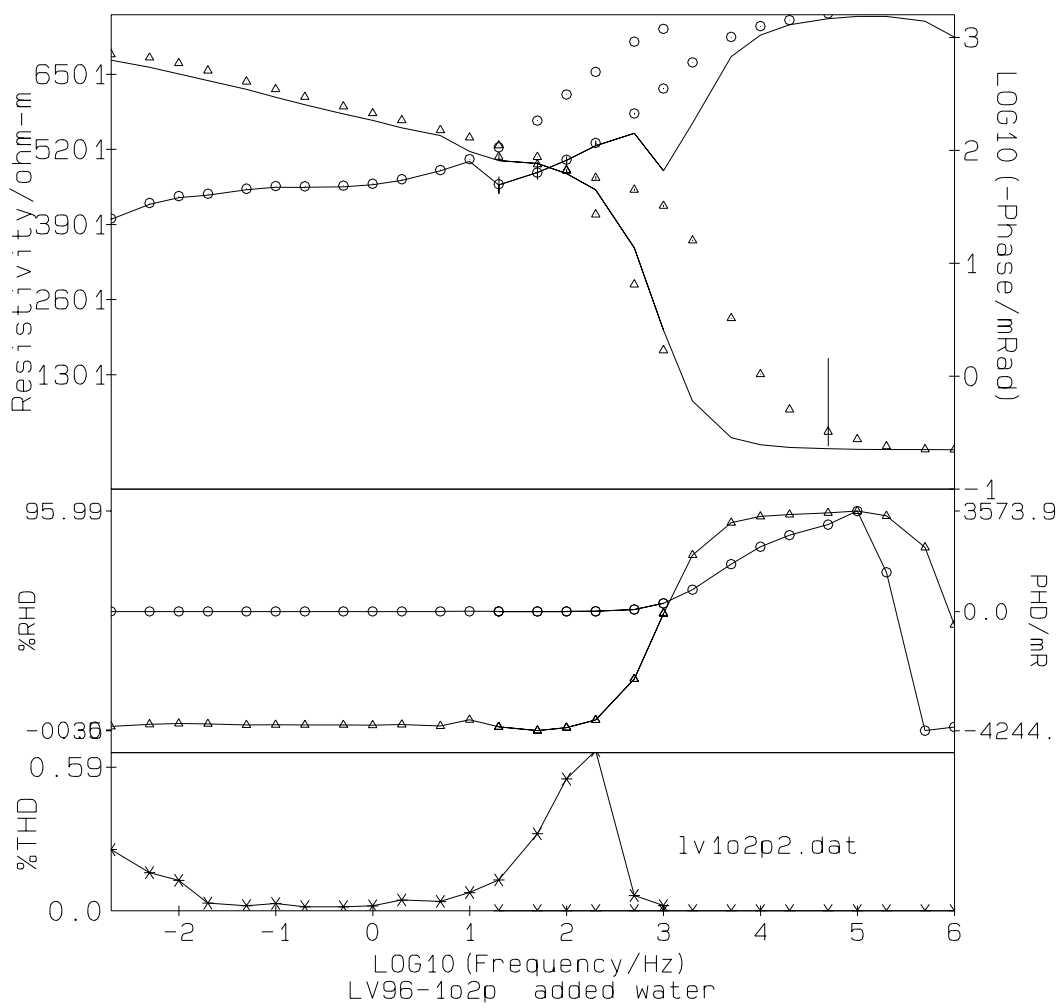

Figure 21. Sample LV-102P. Second measurement, water content increased to $6.6 \%$.

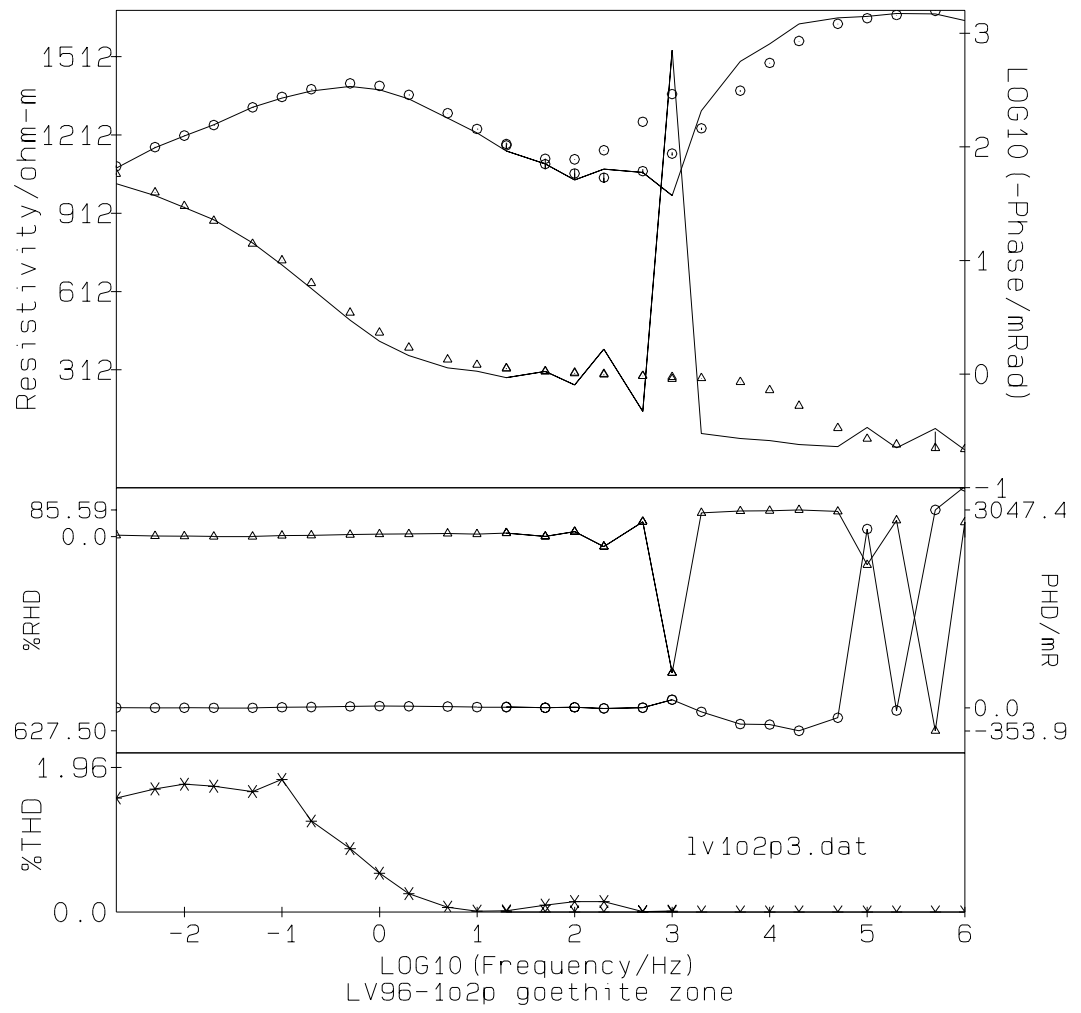

Figure 22. Sample LV-102P. Third measurement, water content increased to $12.1 \%$. 


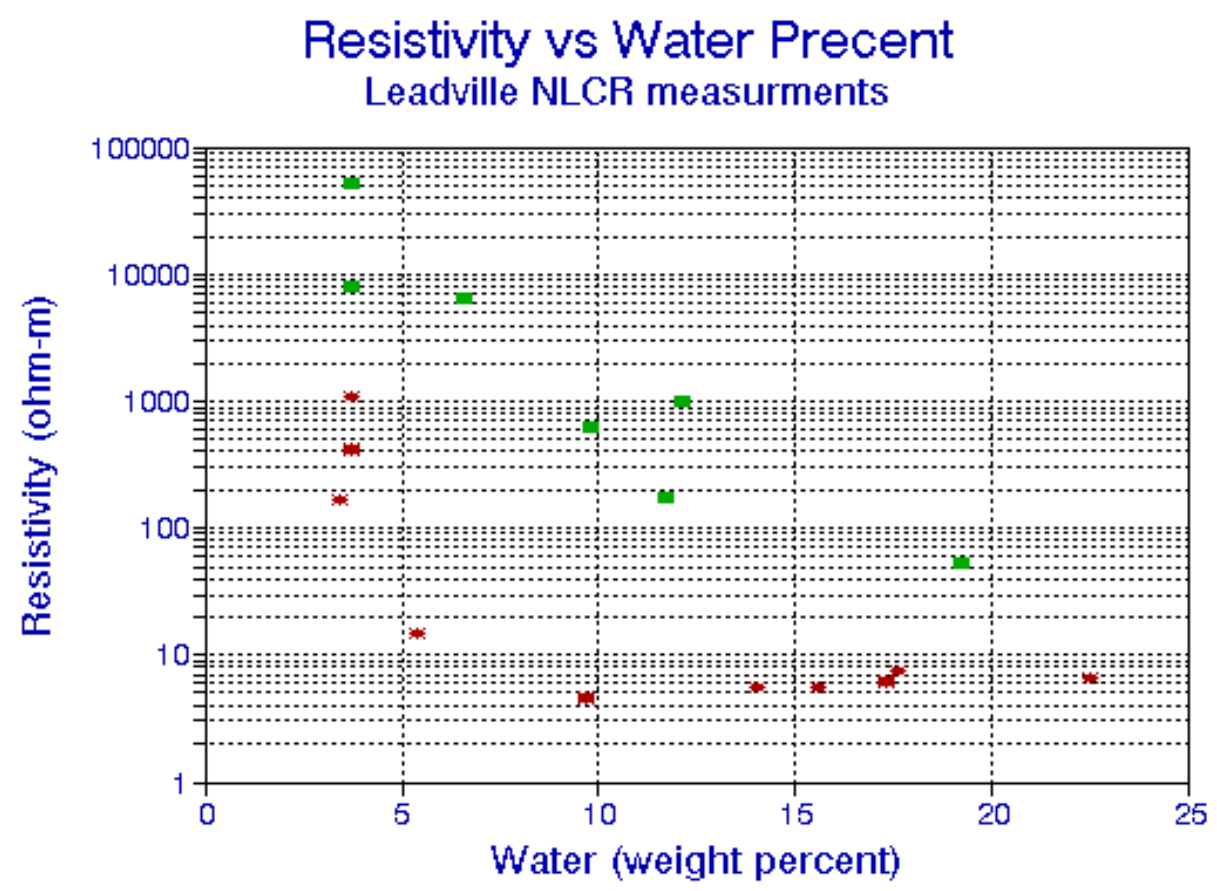

* Jarosite $\square$ Goethite

Figure 23. Plot showing the relationship between resistivity and water content. Jarosite samples show a significantly different relationship than do goethite samples. Slightly above 5 weight percent water, the resistivity of all the jarosite samples drops below $10 \mathrm{ohm}-\mathrm{m}$, whereas the goethite samples show a fairly steady decrease as water content increases. 